\title{
Creative Control: A Neurocognitive Index of Relationships Between Creativity and Attention
}

by

\author{
Naba Ahsan
}

A thesis submitted to the Faculty of Graduate and Post Doctoral Affairs in partial fulfillment of the requirements for the degree of

\section{Master of Cognitive Science}

\author{
Carleton University \\ Ottawa, ON
}

(C) 2016

Naba Ahsan 


\begin{abstract}
The relationship between attention and creativity in task-dependent situations is unclear. Previous work has shown that defocused attention is associated with divergent thinking (i.e. Carson, Peterson, \& Higgins, 2003) and that focused attention is associated with convergent thinking (i.e. Necka, 1999). To address this discrepancy, we assess relationships between early forms of attention (sensory gating) and standardized creative thinking tasks. Attention is indexed by the P50 ERP component. Creative performance is tested in divergent and convergent thinking domains using the ATTA and CRA, respectively, as well as a convergent thinking, non-creative task. We present a correlational analysis between attentional style and creative performance from 22 participants. Our results show that defocused attention enhances divergent thinking, and that focused attention enhances convergent thinking, in both creative and non-creative domains. Furthermore, we demonstrate the utility of the P50 in creativity studies and suggest a methodological contribution that will extend current approaches to extracting P50 values.
\end{abstract}




\section{Acknowledgements}

This thesis is dedicated to the people who mattered most in its compilation. First, I would like to acknowledge my committee for their incredible patience and advisement. I am sincerely grateful to my supervisor, Kasia Mulder, for her constant encouragement and presence throughout this process. Needless to say, this project would not have been possible without her knowledge and dedication, nor would the experience have been as enjoyable. She inspired and motivated me from start to finish.

I am also indebted to Kathy Van Benthem for the countless hours that were so patiently put towards guiding me. It was only with her teachings that I was able to plan and execute this work. Without her input, I might still be unable to distinguish components from noise. She has been an invaluable resource - not only did she point me in the right direction time and again, she never once hesitated to take time out of her busy schedule to do so, and for that I am truly thankful.

To those in ICS who had a positive impact on me over the last two years. A special thanks to Korey MacDougall for putting the time in to ensure the design was exactly as I envisioned, and to Can Mekik for the days spent writing in coffee shops and libraries.

To my family, Ghazala, Shakil, Shuja, and Samaa Ahsan, who instilled in me a love for academia, and who took time out of their own chaotic lives to support me in my passion. And finally, to Abrar Mahmud, who was always there for me, despite the distance, and who put up with me at my worst, all the while pushing me to be my best. 


\section{Contents}

1 Introduction 1

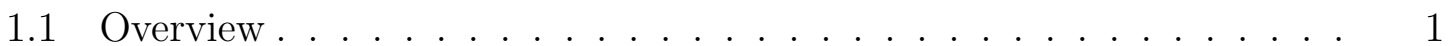

1.2 Research questions . . . . . . . . . . . . . . . . . 3

2 Background I: Neural Foundations 5

2.1 Physiological fundamentals . . . . . . . . . . . . . . . . 5

2.2 Event-Related Potentials (ERP) . . . . . . . . . . . 8

2.2.1 ERP Components . . . . . . . . . . . . . . 9

2.2.2 Auditory Attention Components . . . . . . . . . . . . . . . 9

2.2.2.1 P50 component . . . . . . . . . . . . . . 10

2.2.2.2 N100 component ................ . 11

2.2.2.3 P200 component ................ 12

2.3 EEG equipment set up and recording . . . . . . . . . . . 13

2.3.1 Artifacts and data cleaning . . . . . . . . . . . 13

2.4 Conclusion . . . . . . . . . . . . . . . . . . . . . . . . 14

3 Background II: Attention $\quad 15$

3.1 Attention theories for selective auditory processing . . . . . . . 15

3.1.1 Broadbent: Filter Model . . . . . . . . . . . . . . . 16 
3.1.2 Treisman: Attenuation Model . . . . . . . . . . . . . . 16

3.2 ERP evidence for early selection theories of auditory attention . . . 17

3.3 Conclusion . . . . . . . . . . . . . . . . . . . . . . . . 18

4 Related works: Creativity $\quad 19$

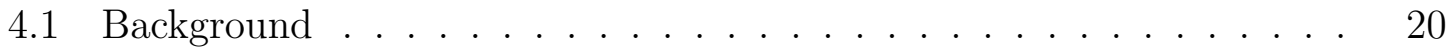

4.2 Attention and creativity associations . . . . . . . . . . 24

4.3 Neuroimaging studies in creativity . . . . . . . . . . . . 28

4.4 Conclusion . . . . . . . . . . . . . . . . . . . . . . . . 31

5 Methods $\quad 32$

5.1 Participants . . . . . . . . . . . . . . . . . 32

5.2 Materials and Apparatus . . . . . . . . . . . . . . . . . . 32

5.2 .1 Cognitive assessment tasks . . . . . . . . . . . . . 33

$5.2 .2 \quad$ EEG equipment . . . . . . . . . . . . . . . 36

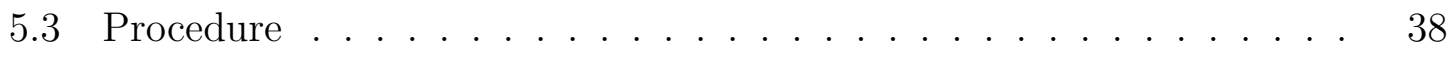

5.3 .1 Preparation . . . . . . . . . . . . . . . . 40

5.3.2 EEG data collection . . . . . . . . . . . . . . . . 41

5.4 EEG data cleaning and pre-processing $\ldots \ldots \ldots \ldots . \ldots 41$

5.4.1 Component filtering and extraction . . . . . . . . . 42

6 Results 44

6.1 Statistical analysis . . . . . . . . . . . . . . 44

6.2 Descriptive data: Divergent and convergent thinking test scores . . . 44

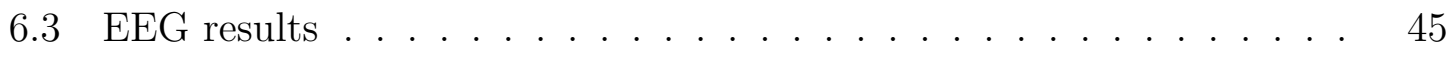

6.3 .1 Manipulation check . . . . . . . . . . . . . . . 45 
6.3.2 P50, N100 and P200 amplitude and suppression values . . . . 47

6.4 Correlational results . . . . . . . . . . . . . . . . . . 47

6.4.1 P50 and divergent thinking ............. . . 47

6.4.2 P50 and convergent thinking . . . . . . . . . . . . 48

6.4.3 N100, P200 and performance scores . . . . . . . . . . 50

6.4.4 Correlations among tasks . . . . . . . . . . . . . . 50

7 Discussion and Conclusion $\quad 53$

7.1 Interpretation of results . . . . . . . . . . . 53

7.1.1 Creative performance and sensory gating . . . . . . . . 53

7.1.2 Non-creative performance and sensory gating . . . . . . . . . 55

7.1.3 Correlations among tasks . . . . . . . . . . . . 56

7.1.4 EEG results: A methodological evaluation . . . . . . . . . 57

7.2 Concluding remarks . . . . . . . . . . . . . . . 57

7.2 .1 Limitations . . . . . . . . . . . . . . . 58

7.2 .2 Future work . . . . . . . . . . . . . . . 59

$\begin{array}{ll}\text { Glossary } & 61\end{array}$

$\begin{array}{ll}\text { Appendices } & 65\end{array}$

$\begin{array}{ll}\text { References } & 69\end{array}$ 


\section{List of Figures}

2.1 Electrical forces exerted by a dipole. . . . . . . . . . . . . . . . . . . 7

2.2 Paired-click design . . . . . . . . . . . . . . . . . . . . . 11

2.3 Sensory gating indexed by the paired-click paradigm . . . . . . . . . . 12

4.1 Mednick's associative hierarchy . . . . . . . . . . . . . . . . 23

5.1 EEG equipment set up . . . . . . . . . . . . . . . . . 37

5.2 Experimental set up: P50 paradigm . . . . . . . . . . . . . 37

5.3 Experimental set up: CRA and control task . . . . . . . . . . . 39

5.4 Typical P50 component . . . . . . . . . . . . . . . . 43

6.1 ERP manipulation check . . . . . . . . . . . . . . . 46

6.2 ATTA (fluency) performance and sensory gating . . . . . . . . . . 48

6.3 CRA performance and sensory gating . . . . . . . . . . . . . 49

6.4 Non-creative performance and sensory gating . . . . . . . . . . . . . 49

6.5 ATTA and CRA performance scores . . . . . . . . . . . . 51

6.6 CRA and control task performance scores . . . . . . . . . . 51

6.7 ATTA and non-creative performance scores . . . . . . . . . . . 52 


\section{List of Tables}

6.1 Performance scores by task . . . . . . . . . . . . . . . . 45

6.2 ERP amplitude values by component . . . . . . . . . . . . . . . 47

6.3 Correlations between N100, P200 components and task performance . 50 


\section{List of Acronyms}

ATTA . . . A Abbreviated Torrance Test for Adults

AUT . . . . A Alternative Uses Task

CAQ .... Creative Achievement Questionnaire

CRA .... Compound Remote Associates

EEG .... Electroencephalography

ERP . . . . Event-Related Potential

EPSP . . . . Excitatory Postsynaptic Potential

HCGSN . . Hydro-Cel Geodesic Sensor Net

IPSP . . . . Inhibitory Postsynaptic Potential

RAT . . . . Remote Associates Test

TTCT ... . Torrance Test of Creative Thinking 


\section{Chapter 1 Introduction}

\subsection{Overview}

Creativity is the ability to produce work that is both novel and useful. Given this definition, it is clear that creativity is needed in endeavours scanning multiple domains, from science, to art, to design. However, what is less clear is our understanding of creativity as a cognitive construct, and thus various studies have investigated this multi-faced phenomenon. Many of these studies have relied on traditional quantitative and/or qualitative methods that measure the key thinking styles associated with creativity. In particular, creative output can be generated by either:

a ) divergent thinking (generation of multiple solution to an open-ended problem), and/or

b ) convergent thinking (generation of a single answer to a closed-ended problem).

Many traditional methods have focused on designing instruments and tasks to measure creativity associated with a given type of thinking. While informative, these approaches do not identify the neurocognitive mechanisms that influence creative outcomes. Accordingly, there has been growing interest in using neuroscience methods to study the neural correlates of creativity (i.e., Arden, Chavez, Grazioplene, \& Jung, 2010; Dietrich \& Kanso, 2010; Srinivasan, 2007). Some have expressed that 
"any theory on creativity must be consistent and integrated with a contemporary understanding of brain function," (Pfenninger \& Shubik, 2001, p. 217). Among the prominent neuroimaging methods in creativity is electroencephalography (EEG), which allows for time-sensitive neural recordings.

While EEG research has taken strides in moving the understanding of creativity forward, the majority of studies do not investigate the various cognitive mechanisms in creative endeavours; one such mechanism is attention, which is known to mediate most (if not all) cognitive constructs. While this mechanism is clearly important for creativity, its role is not yet clear, with some studies showing creativity is enhanced by focused attention and others showing the opposite (creativity is enhanced by defocused attention). To date, few attempts have been made to associate the distinct forms of creative cognition (divergent vs. convergent) with the style of attention that best mediates it. In fact, despite the focus on individual factors, creative ideation has rarely been studied on a task-dependent basis.

In this thesis, we investigate the neurocognitive mechanisms by which attention mediates creativity in divergent and convergent thinking scenarios. Standardized divergent and convergent thinking tasks are used to assess creative and non-creative performance. EEG is used to index focused versus defocused attention styles using a very early form of attention, the P50 component, also known as sensory gating. A correlational analysis examines relationships between task-dependent creative outcomes and attention styles.

Research questions can be found in section 1.2 of this chapter. Chapter 2 delineates the neural foundations to understand EEG recordings, while Chapter 3 provides an overview of relevant attention theories and related concepts. Chapter 4 reviews rel- 
evant theories of creativity, literature on attention and creativity, and concludes with neuroimaging studies in creativity. Chapter 5 presents the methodology, including design set up, stimuli, EEG recording procedure, and data cleaning and pre-processing stages. Chapter 6 presents the results, both behavioural and neurophysiological. Finally, Chapter 7 interprets and discusses the results and concludes with limitations and opportunities for future work.

\subsection{Research questions}

We examine the following research questions:

1. What is the relationship between divergent thinking styles of creativity and early sensory gating?

2. What is the relationship between convergent thinking styles of creativity and early sensory gating?

3. What is the relationship between non-creative, convergent thinking styles and early sensory gating?

Hypotheses related to these questions are based on prior work indicating that divergent thinking is improved given defocused attention or less selective sensory gating (i.e. Carson et al., 2003; Fiore, Schooler, Linville, \& Hasher, 2001) and convergent thinking is improved given focused attention or more selective sensory gating (i.e. Martindale, 1999; Necka, 1999). Thus, in the present work, in terms of divergent thinking a negative correlation between the ATTA and sensory gating is expected (i.e., that defocused attention mediates divergent thinking). On the other hand, a positive correlation between the CRA and sensory gating is expected (i.e., that focused attention mediates convergent thinking). In examining the impact of attention 
as a neurocognitive mechanism in creativity, the intent of this thesis is to contribute to the knowledge of neurocognitive constructs involved in creative endeavours. 


\section{Chapter 2 Background I: Neural Foundations}

The aim of this chapter is to provide background terminology and concepts that will be helpful in processing the remainder of this thesis. I discuss the necessary foundations, including a) the functional basics of EEG recordings at the neurophysiological and instrumental levels, and data extraction, and b) attention theories specific to the direction of this investigation, as well as EEG components that are specific to those attention constructs. The portions most critical to the thesis correspond to ERP components, in particular P50 component.

\subsection{Physiological fundamentals}

The present work investigates the neural signatures of task-dependent creativity using EEG. Studying EEG responses to complex cognitive tasks usually involves recording electrical potentials at the scalp, and then analyzing the resulting data using mathematical principles. It is a basic premise in EEG studies that some of the electrical potentials measured at the scalp originate from neural activity.

At rest, neurons have a resting potential of about $-70 \mu \mathrm{V}$, the result of opposing intracellular and extracellular ionic charges. Permeability of the cell membrane changes in response to an influx of sodium ions and pushes the membrane potential closer to its threshold potential of $-55 \mu \mathrm{V}$. At this point the cell is depolarized and an action 
potential begins, creating a voltage spike that travels from the neuronal cell body, down the axon and to the axon terminal, where neurotransmitters are stored. When a stimulus such as an auditory click is applied, it is received by the corresponding neurons and initiates a series of electric signals, as just described.

EEG records activity mostly from the cortical pyramidal cells, which are neurons with a triangular body and elongated apical dendrites, oriented perpendicular to the cortex. During signal transmission, these neurons produce electric activity primarily through action potentials and postsynaptic potentials (S. Luck, 2014). Postsynaptic potentials are changes in the membrane potential that occur when neurotransmitters are released from the axon terminal of a presynaptic neuron and bind to the dendrites of a postsynaptic neuron.

An action potential may last only a millisecond, but an excitatory postsynaptic potential (EPSP) or an inhibitory postsynaptic potential (IPSP) can last hundreds of milliseconds. The activated network of neurons produces an electric field, a region surrounding the charged particles in which force can be exerted on other ions. In the electric field, the summed voltage of the ion group is great enough to be recorded from a distance. ERP recordings tend to reflect the greater activity of the postsynaptic potentials rather than action potentials themselves (S. Luck, 2014). On the EEG recording, this summed activity appears as a waveform, comprised of underlying components (to be discussed), for which the amplitude values vary between approximately $\pm 100 \mu \mathrm{V}$ (Kuperberg, 2004).

A dipole is created when there are opposing charges separated by a small distance. The dipole is negative at one end, positive at the other end, and approaches zero as the distance decreases and the charges cancel each other out (depicted in Figure 2.1). 


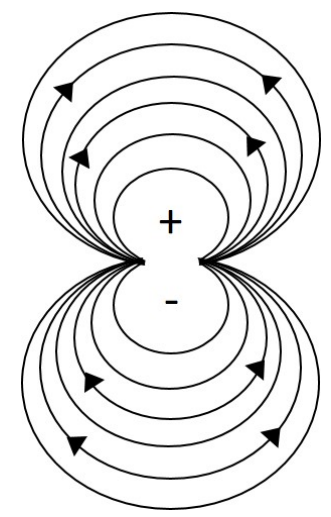

Figure 2.1: Electrical forces exerted by a dipole.

Neurophysiologically, dipoles are speculated to be the results of EPSPs and IPSPs (Dickter \& Kieffaber, 2014). An EPSP depolarizes the membrane (creates a positive change in the resting potential) whereas an IPSP hyperpolarizes the membrane (creates a negative change in the resting potential). This means that an EPSP creates a highly positive environment inside the cell, but results in a highly negative environment outside the cell, and vice versa for an IPSP. Thus, when a large enough dipole results, its equivalent current dipole can be reliably recorded at the scalp (S. Luck \& Kappenman, 2011). From a given dipole, the general spatial distribution of the source can be inferred; the result of the equivalent current dipole will be negative and positive voltage recordings on the scalp, separated by a band of no charge. Normally, the opposing side of the dipole is not noticeable, although with dense array systems (as used in the present work) both ends of the dipole can at times be determined (Delorme, Palmer, Oostenveld, Onton, \& Makeig, 2009). A common misconception, as noted by S. Luck (2014) is that the positivity or negativity of an observed deflection might represent an excitatory or inhibitory neural response. However, because we are unable to decipher the polarity of the neurons from which the activity originates, we 
are unable to extract any further information from the direction of the deflection.

EEG data can be analyzed in frequency-domains (spectral analysis) and timedomains (Event-Related Potentials). Frequency-domain analyses are done via observation of oscillatory activity specific to a stimulus. Decomposing EEG waveforms shows that these oscillations occur within specific frequency distributions which have been associated with cognitive mechanisms and include Delta $(0-4 \mathrm{~Hz})$, Theta (4-8 $\mathrm{Hz})$, Alpha $(8-12 \mathrm{~Hz})$ Beta $(12-30 \mathrm{~Hz})$, and Gamma $(30-80 \mathrm{~Hz})$ (Dickter \& Kieffaber, 2014). Time-domain analyses are a specialized form of EEG recording known as Event-Related Potentials (ERP). An ERP study provides excellent temporal resolution and is the focal methodology employed in the present study. The following section describes ERP studies in more detail.

\subsection{Event-Related Potentials (ERP)}

In an ERP study, specific stimuli in a cognitive task are time-locked to the neural response with millisecond precision (Gaudreault et al., 2013) (other forms of EEG recordings are those which recognize specific frequency waves during a task, but are not time sensitive and will not be discussed here). ERPs are gaining popularity in cognitive neuroscientific research applications. Of all the non-invasive neuroimaging methods, ERP provides the most direct measure of neurocognitive function with the greatest temporal resolution (Kuperberg, 2004).

The most common procedure for ERP studies is presenting the participant with repeated stimuli; responses to these stimuli are then extracted from the overall EEG data by isolating epochs (time windows) surrounding the stimuli response and computing an average waveform, known as a component. This repetition of stimuli and averaging serve a vital function; they help to isolate ERP components. This is because 
any voltage activity that is not time-locked will not appear consistently throughout the epochs (Kuperberg, 2004). Electrical activity that is not of interest, also known as artifacts, can be rejected as it is unrelated to the cognitive processes of interest. Therefore the entire epoch is commonly discarded so as to avoid contaminating data during ERP averaging.

\subsubsection{ERP Components}

One way to distinguish between different kinds of ERP components is by the timing of their occurrence, which splits them into two classes (Landa, Krpoun, Kolarova, \& Kasparek, 2014). Early wave ERPs normally peak within the first 200 milliseconds following stimulus onset and represent "sensory" or "exogenous" filtering (sensitive to the physical nature of the stimulus). Later ERPs are called "cognitive" or "endogenous" (sensitive to the meaning or processing demands of the stimulus).

Within early and late ERPs, waveforms can be further differentiated based on polarity (positive $[\mathrm{P}]$ or negative $[\mathrm{N}]$ ), scalp distribution, and response to task ma-

nipulation (Landa et al., 2014). A standard, operational definition conceptualized by Donchin, Ritter, and McCallum (1978), and revised by S. Luck (2014) is as follows:

An ERP component can be operationally defined as a set of voltage changes that are consistent with a single neural generator site and that systemically vary in amplitude across conditions, time, individuals, and so forth. That is, an ERP component is a source of systematic and reliable variability in an ERP data set.

\subsubsection{Auditory Attention Components}

A number of ERP studies have addressed questions about attention processing, in particular, how attention modulates the flow of sensory processing. Attention is 
unique in that it is a set of processes that control the flow of information through the nervous system; in other words, it is recognized as a system that modulates the functionality of other cognitive operations (i.e. perceptual, memory and response systems), and consequently, that influences most, if not all, ERP components (S. Luck \& Kappenman, 2011). Sensory gating is an important neurocognitive function that is thought to protect the integrity of attention and reflects the ability to attend to salient information and attenuate irrelevant stimuli, thereby optimizing information processing in the brain (Braff \& Geyer, 1990; Light \& Braff, 2003). This pre-attentive mechanism is most commonly indexed by the P50 component, and has recently been shown to have relationships with the N100 and P200 components. This section will focus on the auditory evoked P50, N100, and P200 ERP components as they are specific to auditory sensory gating and early attention processing.

\subsubsection{P50 component}

The P50 is a very early component (S. Luck \& Kappenman, 2011). It is among the least studied components, but is nonetheless important in terms of indexing attention mechanisms. Specifically, it is thought to represent the ability of the neural inhibitory pathway to selectively gate and attenuate irrelevant information from entering the conscious processing stream. This is further evidenced by findings that the P50 is unaffected by attentional manipulations, unlike later attentional components, such as the N100 and P200 (Jerger, Biggins, \& Fein, 1992; Picton \& Hillyard, 1974).

The most common method used to elicit the P50 is the paired-click paradigm (Freedman, Adler, Waldo, Pachtman, \& Franks, 1983) and it is based on the idea that attenuation of irrelevant stimuli in the brain indexes the strength of inhibitory neural mechanisms (i.e. Adler et al., 1982; Sur \& Sinha, 2009). The paradigm 
presents participants with two identical auditory clicks, usually 500ms apart since longer intra-pair intervals result in diminished effect sizes (Adler et al., 1982; Dolu, Süer, \& Özesmi, 2001). Inter-pair intervals typically between 6 - 10s to prevent carry-over effects (i.e. recordings of cortical activity from the previous stimuli) (see Figure2.2). (i.e. Dalecki, Johnstone, \& Croft, 2015; Zouridakis \& Boutros, 1992).

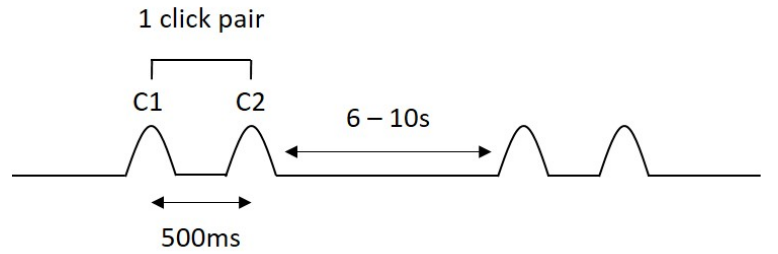

Figure 2.2: Overview of the P50 paired-click paradigm. Intra-pair click intervals are presented 500ms apart, with inter-pair intervals set between 6 - 10 seconds.

Response suppression at the scalp is indexed for the second click (C2) following the identical first click $(\mathrm{C} 1)$. Peak amplitude values are typically extracted at the vertex electrode $(\mathrm{Cz})$ between 30 - 80ms and averaged across $\mathrm{C} 2$ and $\mathrm{C} 1$. Difference scores are calculated by subtracting the peak amplitude value of the second click from the peak value of the first click. Larger difference scores represent stronger sensory gating, indicating a more focused attentional style (see Figure 2.3). To the best of our knowledge, the P50 component has been employed only once before in creativity research by Zabelina, O'Leary, Pornpattananangkul, Nusslock, and Beeman (2015). The study presented here will be the second to employ the component, and the first to correlate it with other forms of creative thinking.

\subsubsection{N100 component}

The N100 component is a negative deflection observed 100ms following auditory stimulus onset. It normally indicates the detection of acoustic changes in the environment, 
A)

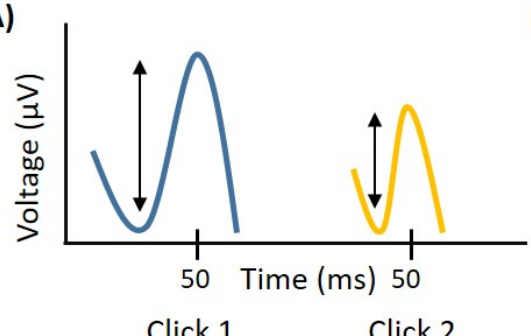

B)

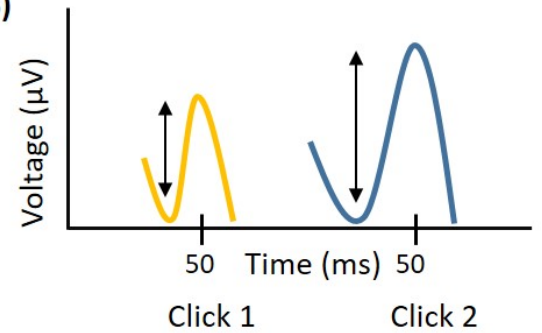

Figure 2.3: (A) Decreased response to Click 2 vs. Click 1 indicates more focused attention, whereas (B) an increased or equal response to Click 2 vs. Click 1 reflects defocused attention.

both as the result of frequency changes in stimuli and sound onset. It is extracted between 80 - 150ms. This is considered an "obligatory cognitive matching process"; when a stimulus is presented, it is neurologically matched with the preceding stimuli (Sur \& Sinha, 2009). The N100 can be a reliable measure of sensory gating, but only when no other attentional manipulations are present within the study (Guterman, Josiassen, \& Bashore, 1992; Smith, Boutros, \& Schwarzkopf, 1994). However, it likely represents different attentional functions than the P50; in particular, N100 gating could reflect the triggering of attention (Näätänen \& Picton, 1987), perhaps even as a function of working memory (Lijffijt et al., 2009).

\subsubsection{P200 component}

The P200 is a positive deflection observed 200ms after stimulus onset, and the peak is normally extracted between 150 - 250ms. It is modulated by several cognitive functions including ageing and is often employed in infant and child studies regarding feature-based attention (S. J. Luck \& Hillyard, 1994), although further work is required in this area. The P200 has only been implicated in sensory gating studies thrice. Boutros, Korzyukov, Jansen, Feingold, and Bell (2004) found that P200 
gating correlated with P300 amplitude and latency in an oddball task ${ }^{1}$; Zabelina et al. (2015) found no associations between the P200 and P50 amplitudes, and performance on creative assessments; and, Lijffijt et al. (2009) suggested that the P200 reflects allocation of attention and the initial conscious awareness of a stimuli.

\subsection{EEG equipment set up and recording}

An EEG net consists of electrodes (channels) that are placed on the scalp in specific positions that correspond with the International 10-20 system. A conductive fluid is needed at the junction of the scalps conductive tissues and the metal or sponge of the electrode (see Dickter and Kieffaber for details). Depending on the EEG style, this will be in the form of commercial EEG gels or mixable solutions. In the present study, we use a 128-channel HydroCel Geodesic Sensor Net with sponge electrodes and a liquid electrolyte solution.

\subsubsection{Artifacts and data cleaning}

During the experimental session, the EEG recording is susceptible to noises in the environment. Noise can show up as voltage spikes in the data but it needs to be accounted for since it is not directly related to cognitive processing. These non-cognitive spikes are known as "artifacts" and may be mechanical or instrumental (such as an added electric current from a computer or cell phone), environmental (such as noise produced from an overhead fan or footsteps) and physiological (such as an eye movement or a heartbeat). It is important to account for this during testing; participants should be asked to minimize unnecessary movement and noise and electrical devices should be eliminated as much as possible. Filters can be applied based on the range in

\footnotetext{
${ }^{1}$ In an auditory ERP oddball task, repetitive stimuli are interspersed with deviant tones.
} 
which the processing occurs. Band-pass filters selectively pass only the signals within a certain range of frequencies and reject data outside the parameters, whereas notch filters will remove all data within the specified frequencies. These frequency ranges are usually recommended by previous studies that have validated the use of certain parameters with respect to obtaining the cleanest data for the component.

\subsection{Conclusion}

This chapter has discussed neural and instrumental foundations as they relate to the present thesis direction, in which we index early sensory gating with the P50 ERP component. The following chapter provides a background on the theoretical and neural mechanisms of early selection forms of attention. 


\section{Chapter 3 Background II: Attention}

Attention theories agree that humans have a limited capacity for attention. Cognitive mechanisms cater to this limitation by selecting or discarding information before it enters the conscious stream for further processing (Kahneman, 1973). Among the most prominent theories that view attention as a limited cognitive resource are bottleneck theories, which assume that attentional selection occurs early, before semantic content is processed. As per the name, bottleneck theories suggest the presence of cognitive mechanisms that restrict the flow of information by selectively attending to certain stimuli and ignoring others. These theories can be applied to both visual and auditory domains, but as the present work focuses on early auditory processing, the following section explains prominent bottleneck theories in this light.

\subsection{Attention theories for selective auditory processing}

Donald Broadbent and Anne Treisman proposed highly influential bottleneck theories of attention (Mikyska, 2012); interestingly, ERP studies investigating early, selective attention provide physiological support for these psychological theories by showing that a) ERP auditory components peak at pre-conscious levels and b) information is selected based on physical features. Recall that early selection mechanisms can

be studied via the P50, N100 and P200 components. Broadbent's and Treisman's 
models will be discussed, followed by supporting ERP data.

\subsubsection{Broadbent: Filter Model}

The first bottleneck theory, Broadbent (1958)'s Filter Model, proposes that prior to information processing, auditory stimuli pass through a selective filter which evaluates the physical characteristics of the stimuli (i.e. location, pitch). Stimuli that match the demands of the filter are attended to, while unselected stimuli are filtered out. Broadbent demonstrated this using the dichotic listening test that presents both ears with auditory information simultaneously and asks the participant to repeat information from attended versus unattended ears, or as Broadbent referred to them, channels. Broadbent found that people can only attend to information from one channel at a time and that because this early selection is done on a purely physical feature basis, semantic processing of information occurs at a much later stage.

\subsubsection{Treisman: Attenuation Model}

The Filter Model was revised by Treisman (1964) based on the finding that unattended information is still sometimes processed, such as hearing ones name across the

room. Her Attenuation Model suggests that unattended information is attenuated, but not completely discarded as in the filter model. Stimuli are selected for further processing when they reach an intensity threshold. The threshold is systemically determined starting with physical characteristics and moving up to semantics. Certain words or concepts maintain a very low threshold, such as ones name or danger words like "fire". 


\subsection{ERP evidence for early selection theories of auditory attention}

ERP studies of early selection provide support that attention can be pre-consciously assessed for rejection or processing value. Hillyard, Hink, Schwent, and Picton (1973) developed a selective listening task in which he presented participants with binaural tones and had them respond. Participants either responded to stimuli in one ear only (condition 1) or in both ears (condition 2). Hillyard et al. (1973) recorded taskcontrolled ERPs when stimuli were ignored versus attended to. He found that the N100 component was greater for attended stimuli, suggesting that the N100 is an early sensory gating mechanism that filters out irrelevant stimuli.

In a follow up study, Näätänen and Picton (1987) proposed that the differential negativity in the N100 waves Hillyard observed could be a result of the auditory

cortex comparing features of the initial to incoming stimuli. A trace is created based on early physical features (i.e. tone, pitch, etc.), to which subsequent stimuli are compared to and either rejected or selected for further processing. This occurs at a very early stage of sensory input.

Woldorff, Hansen, and Hillyard (1987) further employed the dichotic listening task, and found differences in P50 amplitudes based on attentiveness versus inattentiveness to sounds in either ear. The study was later successfully replicated (Woldorff \& Hillyard, 1991), overall providing support for the early selection hypothesis of attention by showing that the difference in P50 values during attentiveness and inattentiveness were indeed due to filtering mechanisms and not attributable to carry-over effects or noise. 


\subsection{Conclusion}

As per the studies mentioned, ERP data adheres to the early selection theories of attention. Task-related fluctuations of the N100 and P50 components demonstrate that incoming stimuli can be discarded on the basis of irrelevancy at very early stages, thereby protecting the neural and cognitive processing streams from information overload. In line with the direction of this thesis, it should hold that attention style, as indexed by the P50 component, influences creative cognition. However, very little neurocognitive research focuses specifically on attention as it relates to creativity. In the following chapter, to illustrate the state of the art in creativity research, I will describe both general studies of creativity using EEG methods, as well as ones targeting attention in particular. 


\section{Chapter 4 Related works: Creativity}

Creative cognition has been studied through a variety of research avenues (Sternberg, 2005). It has often been conceived of as a unique process; Gardner (1983), for example, constructed his theory of multiple intelligences on the basis that the mind is modular, and that creative minds rely on a different intelligence than average minds do. Others have argued for a less glamorous perspective, suggesting that creativity is not a unique process, but the compilation of ordinary processes that result in extraordinary outcomes (i.e. Weisberg, 1986, 1999). Most recent views suggest that flexibility in cognitive constructs, such as ones related to knowledge, personality and motivation, can lead to creative output (i.e. Amabile, 1996; Sternberg \& Lubart, 1995; Zabelina \& Robinson, 2010). An important contributing factor to this flexibility is attention, which allocates and mediates cognitive resources: Zabelina and Robinson (2010) note a direct link between mediation of cognitive control and creative thinking. The relationship between attention and creativity, however, remains unclear. As will be discussed below, there is evidence that various forms of attention contribute to creativity; less evidence points to what kind of attention best does so, and whether it is task-dependent. The latter portion of this chapter reviews literature concerning attention-creativity relationships. First, however, we begin with a brief history of the research and theories of creativity. 


\subsection{Background}

The study of creativity reportedly began with Guilford (1950)'s Presidential Address in which he called the neglectful study of creativity "appalling" and stressed that one approach should be to consider the influence of individual differences in creative situations. In outlining possible assessment avenues, Guilford suggested incorporating the following factors: fluency, novelty, flexibility, synthesizing and analyzing ability, complexity, and evaluation. Fluency refers to the ability to generate a large number of ideas in a short amount of time. These ideas need not be original or unique on their own, but the greater number produced, the higher the chance of producing a creative one. Fluency may be tested by asking one to name as many objects as s/he can with a specific property, or to generate titles for a story. Novelty measures the uniqueness of an idea in relation to the norm and can be assessed by word-association tasks. Flexibility is essentially "thinking outside the box" and can be tested by giving the participant a problem that requires a novel approach; for example, the paper must be folded to complete the problem, but the participant must resolve this on his/her own. Synthesizing and analyzing abilities involve organizing smaller ideas into broader ones, or deconstructing ideas to investigate the smaller parts. Complexity is the ability to mentally retain interrelated factors of the conceptual structure of the problem and to resolve these simultaneously. Finally, with evaluation comes the application of the creative work in which the individual must assess the appropriateness of the product within context and this may be done by having him/her score solutions based on excellence and fitness. These factors are still incorporated by creativity researchers in generating psychometric tasks, some of which will be discussed further.

In a subsequent address, Guilford differentiated between two distinct processing 
styles that lead to creative ideation: divergent and convergent thinking. Divergent thinking involves generating multiple, unique solutions to an open-ended problem. For example, the Alternative Uses Task (AUT) developed by Guilford asks participants to come up with various unique uses for common, everyday objects, such as a paperclip. Given that generating a wide range of solutions requires making connections between possibly unrelated concepts, divergent thinking is associated with defocused thinking (Dorfman \& Gassimova, 2015). Since divergent thinking encompasses the assessment factors Guilford suggested, such as fluency and originality, it quickly became a widely used approach to studying creativity (Glover, Reynolds, \& Ronning, 1989) and has been the basis of many standardized tests of creative thinking. The Torrance Test of Creative Thinking (TTCT) is an example of a standardized and widely implemented test of creativity that focuses heavily on items requiring divergent thinking. While the TTCT does not encompass all dimensions of creativity, it is nonetheless a recognized and validated assessment instrument (i.e. Kim, 2006). Since its foundation, several variations have been created one is the shortened Abbreviated Torrance Test for Adults (ATTA). The ATTA is scored on fluency, flexibility, originality and elaboration and involves one verbal and two figural tasks. These aspects are further described in Chapter 5: Methods.

In contrast to divergent thinking, convergent thinking is the generation of a single, correct answer to a closed-ended problem (i.e. one that does not afford multiple solutions). Whereas divergent thinking employs defocused and abstract thinking, convergent thinking is associated with focused and systematic thinking, such as that required by algebraic problems that have a single correct solution and that are solved using a series of logical steps. Insight is a type of convergent thinking task that has 
gained popularity in creativity studies (Finke, 1995). Insight problems are defined by the colloquial "Aha!" moment people have after finding the solution to a problem that seemed unsolvable. An insight problem may appear to be a regular problem, but the solver will hit a mental impasse during which s/he feels all possible solutions have failed. At this point, a hint or incubation time leads to a restructuring of the problem, the solver is able to interpret the problem in a new light, and the solution appears almost suddenly, leading to the "Aha!" moment.

Word-association tasks are a specific type of insight problem used to assess convergent thinking styles of creativity. Mednick (1962)'s Remote Associates Test (RAT) is a standardized test in this domain - each item of this test presents three stimuli words such as cream/skate/ water and is asked to come up with a fourth word that links the three. Here, the answer is ice (ice cream/ice skate/ ice water).

The RAT is derived from Mednick's associative theory of creativity. This theory is based on the hierarchical organization of concepts and the relationship of this organizational structure to creativity. Specifically, mental concepts, once learned, are all associated with one another to some degree depending on the closeness of those concepts in the real world. For example, the concept of daffodil will be more closely associated with yellow than with dog. Thus, individuals should show probabilistically similar responses to cue words (Martindale, 1999). Individuals with higher creative potential are said to show flatter associative hierarchies, whereas lesser creative individuals will show steeper hierarchies (Mednick, 1962). Figure 4.1 shows a possible association hierarchy for the word table. A more conventional association would be chair, indicating a steep associative hierarchy given that the most common conceptual representation is also the most dominant. With a flatter hierarchy, a creative 
individual may still retrieve concepts such as cloth and wood, but is able to surpass conventional responses and retrieve more tangential concepts as well, such as food. As Mednick suggests, "the word association behaviour of the high creative individual should be characterized by less stereotypy and commonality".

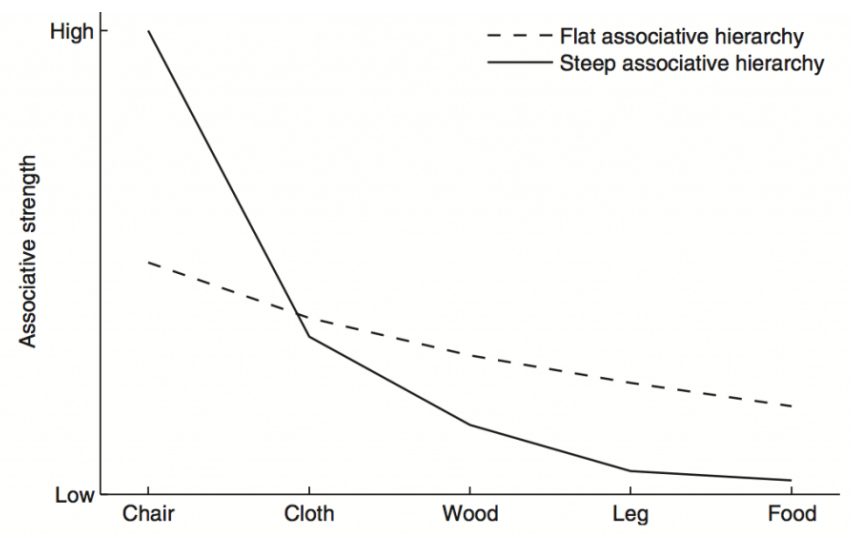

Figure 4.1: Mednick's associative hierarchy for the word "table". Creative thinking is characterized by flatter hierarchies that allow for the retrieval of more tangetial associations. Retrieved from Benedek and Neubauer (2013), p. 274.

Accordingly, it seems that creative individuals tend to be those who are better able to mediate between competing cognitive processes (Fink, Grabner, et al., 2009). We have discussed Guilfords divergent and convergent thinking processes, and Kahneman (1973)'s dual-system approach. Along these lines, Kris (1952) proposed that creativity was a function of competing primary and secondary thought processes. Primary processes dominate the states of dreaming or reverie and "tend to operate on concrete images rather than abstract concepts," whereas secondary processes occur during the waking, conscious states and are "logical [and] reality-oriented." Creative potential is positively associated with the ability to navigate between the two, delving into the primary process to dismiss cognitive boundaries, and bringing those abstract thoughts into consciousness through the secondary process. Less creative individuals may find 
themselves "stuck" within the primary process, therefore unable to "create".

So why do some individuals find themselves unable to create? The paradox in studying creativity is that even though the most creative ideas are new and original, they must still be derived from the pre-existing concepts, episodes or events that are stored as memories. Existing research has explored various factors that influence creative outcomes, including attentional styles (i.e. Mendelsohn, 1976), personality traits such as introversion and extraversion (i.e. Kumar, 1978; MacKinnon, 1970; Tapasak, Roodin, \& Vaught, 1978), intelligence (i.e. Barron \& Harrington, 1981; Feist \& Barron, 2003; B. Hennessey, 2004; B. A. Hennessey \& Amabile, 2010; Runco \& Albert, 1985; Winner, 2000), and affective states (i.e. Aspinwall, 1998; Fredrickson, 2001; Friedman, Förster, \& Denzler, 2007; Isen \& Reeve, 2005; Kaufmann, 2003; Martin, Ward, Achee, \& Wyer, 1993; Simonton, 1999; Vosburg, 1998). Here, I outline work that is most aligned with the present thesis direction, namely attention.

\subsection{Attention and creativity associations}

As the theories outlined above suggest, attention is a strong mediator of the cognitive processes that give rise to creative output. However, what is less clear is what type of attention best mediates creativity. In particular, creative individuals have been associated with both focused and defocused (i.e., leaky) types of attention (Zabelina \& Robinson, 2010), making the role of attention unclear. One example of this comes from studies involving the same individual, such as creative scientists who reported feeling disconnected in their attention prior to a moment of insight, but a focused state following it (Ghiselin, Rompel, \& Taylor, 1964). The following section surveys studies that found leaky attention aids creativity, focusing first on research outside of neurocognitive science. 
Many historical creative individuals were documented for having leaky attention and taking extreme measures to reduce environmental distraction, from Marcel Proust asking servants to tiptoe around the house, to Chekhov calling the sound of his father reading aloud in the next room "abominable conditions" (Kasof, 1997). Mendelsohn (1976) and colleagues argued that creativity is facilitated by a wide breadth of attention, also known as defocused attention. Attention breadth is widened when there are a greater number of stimuli to focus on; thus, if "focused attention captures relevant information [and] defocused attention extends to both relevant and irrelevant information, focused attention is narrowing as compared to defocused attention, which is widening" (Jauk, Benedek, \& Neubauer, 2012).

In a series of studies demonstrating that creative individuals are more sensitive to distracting and irrelevant stimuli, Mendelsohn (1976) manipulated attentional states on the RAT by disclosing to one group they would receive extra information, but not to the other group. The former group divided their attention between the problem and the new information, while the latter focused their attention entirely on the problem. The divided attention group scored higher on the RAT, leading Mendelsohn to argue that creativity is associated with less focused attention. In Mendelsohn and Griswold (1964)'s study, participants were given a distracting list of 25 words to memorize, while another 25 words played on a tape recorder. Unbeknownst to the participants, ten words from each list contained solutions for the anagram task. The authors predicted individuals with higher creative abilities would show a greater inability to filter out distracting information which would be represented by better performance on the anagram task. Creativity was assessed using the RAT and indeed, higher RAT performance correlated with a greater number of solved anagrams, indi- 
cating a higher inability to filter out information. Mendelsohn and Lindholm (1972) built on this by giving participants the option to attend broadly or narrowly to stimuli by asking them to memorize sets of 25 words from two different sources simultaneously (broad attention group), or separately (narrow attention group). Similar to the previous study, each list of 25 words contained ten hints. When asked to recall the words, in contrast to the narrow attention group, the broad attention participants rarely confused the words. The broad attention group also performed higher on the anagram and RAT, once again establishing a link between defocused attention style and creative performance.

The above studies suggest that individuals with higher creative capacity have defocused attention and "cognitive disinhibition" (Eysenck, 1995) that allows them to generate a greater variety of novel and original combinations. Indeed, creative individuals have shown a tendency to self-report as being distractible (Domino, 1970; Powers, Stevens, \& Andreasen, 1975); Kasof (1997) further found that creativity correlated positively with a broad attention span indexed using a self-report measure of attention. Along these lines, Rawlings (1985) found that creative individuals do not display effective attentional filter mechanisms: creative performance correlated with increased errors in a dichotic listening task.

In general, much of the creativity research focuses on the relationship between creativity and defocused attention (Dorfman \& Gassimova, 2015). However, creativity has also been linked to focused attention. For example, Groborz and Necka (2003) found that higher performance on a non-creative number generation task, which requires focused attention, correlated with enhanced performance on a standard creative thinking-drawing task. Necka (1999) tested individuals in dual-task 
situations, which require more focused attention styles to mediate between two different activities. Creative individuals performed poorly compared to their less creative counterparts. Colzato, Ozturk, and Hommel (2012) had participants undergo focusedattention meditation that promoted cognitive control and concentration on a single thought. This intervention enhanced creative divergent thinking, but constrained convergent thinking.

The variability in findings, i.e., that creativity is associated with various types of attention, strongly alludes to two notions. First, to that of cognitive competition, mentioned earlier through the theories of Guilford, Kahneman, and Kris. Attention is evidently a mediator of cognitive resources, all forms of which can enhance creative performance in the right setting. This leads to the second notion that the nature of attentional influence in creativity is task-dependent. Zabelina and Robinson (2010) demonstrate this in a study that assessed creativity with standardized tests, and cognitive control by the Stroop task. Creative individuals showed greater modulation of cognitive control demonstrated by higher performance on a trial-by-trial basis. The authors conclude that flexibility in cognitive control (i.e. employing more or less focused attention) benefits creativity, and is enhanced when the form of attention pertains to the nature of the task. What the current research lacks at this point is an understanding of how attentional styles relate to different forms of creative thinking. Specifically, are convergent thinking styles of creativity associated with more focused or defocused attention? What about divergent thinking styles?

To answer these questions, we turn to neuroimaging methods of investigating creativity. As will become evident, some more recent creativity work has taken a neuroscientific approach. Popular in this domain is the electroencephalograph (EEG) 
as it allows for time-sensitive data collection. This methodology can be used to index various attentional and creativity styles, as will be reviewed in the following sections.

\subsection{Neuroimaging studies in creativity}

Dietrich and Kanso (2010) and Arden et al. (2010) present extensive reviews outlining neuroscientific findings in creativity. As that review highlights, much of the EEGrelated work in creativity involves spectral analysis of EEG frequency data with a focus on changes in the alpha frequency (see Fink \& Benedek, 2014), which is thought to represent cortical inhibition, leading to a defocused, abstract state of mind. Higher alpha has been associated with overall enhanced creative performance (i.e. Martindale \& Hasenfus, 1978; Martindale \& Hines, 1975; Martindale, Hines, Mitchell, \& Covello, 1984), generation of original responses (Grabner, Fink, \& Neubauer, 2007), and creativity in expertise related situations (i.e. Fink, Graif, \& Neubauer, 2009). These recent findings suggest that alpha increases as a function of creative demands (i.e. Fink, Schwab, \& Papousek, 2011; Razumnikova, 2007) or as a result of creativity training (Benedek, Fink, \& Neubauer, 2006; Fink \& Neubauer, 2006).

However, the approach of correlating alpha with creative performance has some limitations. Klimesch (1999) and Klimesch, Sauseng, Hanslmayr, Gruber, and Freunberger (2007) demonstrated that alpha relates to information processing as a whole (not specific to creativity). Furthermore, while the above-cited studies have provided indications of some of the neural aspects related to creativity, they have also been critiqued for not relying on standardized creativity tasks. In particular, most researchers employ homegrown measures of creativity, such as imaginative or story-telling tasks, which may reduce reliability of the results (Arden et al., 2010). A final limitation is that frequency approaches tell us little about the fine-grained cognitive mecha- 
nisms associated with creativity, something that ERP methods have the potential to remedy.

We now turn to studies that employ ERP methods for studying creativity, which is also the focus of the current work. ERP methods enable us to investigate the neural components of creative performance, thereby closing in on the links between cognitive processes and creativity. In contrast to the present thesis that investigates attentional mechanisms on various creative tasks, the majority of ERP studies to date have focused on convergent thinking insight tasks in the creative domain. The number of these studies is still small: at the time of their review, Dietrich and Kanso (2010) reported six ERP studies in the creativity literature. ERP studies since then have been sparse still, and have not necessarily employed standard tests of creativity. For example, Rutter et al. (2012) examined the N400 component in a semantic processing task that assessed conceptual expansion in creative thought. The N400 amplitudes were greatest during readings of novel and unique sentences, attesting to the usability of ERP methods in creativity studies. Furthermore, ERP provides the benefit of time-locking stimuli to neural responses, which EEG frequency data does not offer. Perhaps for this reason, much greater consistency has been observed with results in ERP domains than with EEG. Convergent thinking task studies of creativity are reviewed next.

A variety of ERP components have been employed in the study of creativity, mostly in the later time ranges post stimuli onset. The P300 component has been found to be affected by distractions during regular problem solving, but not during insight tasks, suggesting that insight thinking requires less planning and working memory (Lavric, Forstmeier, \& Rippon, 2000). Qiu and colleagues (2006; 2008) con- 
ducted a series of studies using convergent tasks in which the figurative manipulation of a Chinese character is the solution for a riddle. They found that N320 amplitudes (thought to reflect reappraisal or later cognitive processing) were greater for resolved versus unresolved insight trials (study 1). Also, amplitudes between 200-600ms were greater for successful (aha) versus unsuccessful (no-aha) solutions (study 2). Lang et al. (2006) found that overall ERP activity appeared different for individuals who gained insight during problem solving compared to those who did not. In two studies, ERP activity was localized at the anterior cingulate cortex (ACC) during aha moments, a neural structure that has previously been implicated in insight solving (Mai, Luo, Wu, \& Luo, 2004; Qiu, Li, Jou, Wu, \& Zhang, 2008)

Most recently, Zabelina et al. (2015) have taken an important step in creativity research by investigating process-specific but task-dependent factors that mediate creativity. The authors investigated the impact of early attention gating on creative performance. Attention gating was indexed by the early P50 component, an indicator of the individuals ability to suppress irrelevant information. Addressing the earlier critiques related to lack of task standardization, participants were assessed on their creative abilities using two standard measures: the ATTA, a divergent thinking test of creativity, and the $\mathrm{CAQ}^{1}$, a real-world creative achievement questionnaire. Results showed that divergent thinking performance correlated negatively with their measure of sensory gating (indicating more focused attention), but that CAQ scores correlated positively with sensory gating. Thus, Zabelina et al. (2015) concluded that creative task interacts with attentional style).

The above mentioned research is an important step in highlighting the variable

\footnotetext{
${ }^{1}$ The Creative Achievement Questionnaire (CAQ) is a standard measure of real-world creative achievement.
} 
nature of attentional influence in creative performance, but more work is needed before a full understanding is reached. First, since to the best of our knowledge this is the only study in this area, it requires further investigation and replication. Moreover, Zabelina et al. (2015) did not study the influence of attention on creative convergent thinking.

\subsection{Conclusion}

Investigating the impact of attention in creativity is important for a number of reasons. First, from a theoretical perspective, doing so adds to the community's knowledge of the cognitive factors that impact creative endeavours. There may also be practical applications in the future. Fostering creativity through various interventions is an active measure being taken in various societal domains. However, the prerequisite for fostering creativity is understanding the influence of cognitive factors

that mediate creativity, one of which is attention. Establishing links between creative cognitive styles and attention forms is the first step in promoting creativity. 


\section{Chapter 5 Methods}

\subsection{Participants}

The study participants were 36 Carleton University students $(M$ age $=21.06, S D$

$=4.31 ;$ Female $=18$ ). Individuals were recruited from Carleton University's undergraduate research site (SONA), and were given two percent bonus course credit as compensation. To participate, individuals had to be 18 years of age or older and fluent in English.

\subsection{Materials and Apparatus}

The study involved two phases. The first phase involved three cognitive assessment tasks:

1. the ATTA, a divergent thinking test of creativity,

2. the CRA, a convergent thinking test of creativity, and

3. a non-creative, convergent thinking task.

The second phase involved an EEG session. 


\subsubsection{Cognitive assessment tasks}

\section{Divergent thinking task}

The Abbreviated Torrance Test for Adults (ATTA) is a shortened and alternate form of the standardized TTCT Goff and Torrance (2002) that assesses the creative facets of originality (producing uncommon, novel or unique ideas), fluency (producing a number of ideas relevant to the task), elaboration (embellishing ideas with details) and flexibility (processing the same stimulus in different ways). The ATTA includes one verbal and two figural tasks. The participant is given three minutes to respond to each of the three tasks. The verbal task corresponds to a single open-ended problem that asks participants to generate multiple unique responses. The two figural tasks have the participant draw novel concepts using incomplete figures as starting points. To guide participants through the tasks, we followed verbatim the instructions provided in the ATTA manual (Appendix A provides an example). We also followed the scoring procedure outlined in the manual, where each task is allotted a raw score that is subsequently converted to a scaled score to allow for direct comparison across abilities (details are listed in Appendix B). In the upcoming analysis, we assessed creativity using the overall score (raw + scaled) as well as the individual raw scores for fluency and originality (Zabelina et al., 2015). Fluency and originality can be compared using raw scores because the same number of activities contribute to their scores. However, elaboration and flexibility use different number of activities for their raw scores, so to compare between all these, the scores need to be scaled. Creativity index is obtained by summing the total scaled scores plus the creativity indicators (Appendix B). 


\section{Convergent thinking task}

The Compound Remote Associates (CRA) task we used in our study is a modified version of the RAT (see Related Works: Creativity) developed by (Bowden, Jungbeeman, Fleck, \& Kounios, 2005) to test convergent thinking methods of creativity with neuroimaging methods. The leading difference between the RAT and the CRA is that the CRA requires compound word associations. The CRA presents the participant with three seemingly unrelated words (i.e. Swiss/ brick/ cottage) and asking them to come up with a single word (cheese) that links with each of the words to create three separate, familiar compound words or phrases (Swiss cheese/ brick cheese/ cottage cheese). The RAT solutions may or may not form compound words; the goal is simply an associative word. A RAT example is falling/ dust/ actor for which the solution word is star. While falling star and stardust are compound words, star actor is not; rather, the words star and actor are simply associated in terms of fame and movies. In short, the CRA represents a subcomponent of the RAT. Bowden et al. (2005) note the following advantages of the CRA in neuroimaging studies: 1) the problems are short and can be solved quickly so that multiple trials can be administered during a session (as stated in Chapter 2, multiple trials are a necessary component of ERP studies), 2) solution words are single-word and unambiguous, simplifying the scoring process, and 3) they are physically compact, making it easier to display the entire trial on a screen, thereby eliminating the need for the participant to move their head or scroll through text.

A single CRA trial corresponds to a triplet made up of three words. In our study, the trials were organized into blocks; as described in the procedure (below), there were two blocks of CRA trials. To design the set of trials for a given block, we relied on 
Bowden and Jung-Beeman (2003)'s study in which they assessed timing and accuracy norms for $144 \mathrm{CRA}$ word triplets. We imposed a 15-second time limit for providing a response for each CRA trial. This limit provides a balance between allowing some time to search for the solution without slipping into a prolonged trial-and-error method. Furthermore, this limit ensures participants would not bore too quickly with the task. Based on this limit, we estimated the number of trials we would need to prepare for each five-minute block, given that there would be variability in participants' ability to solve the tasks, as follows. At the very least, we needed 20 trials in the case that a participant was unable to solve any of the associations. At most, we generously predicted 45 trials per block, in the case a participant was able to solve each trial in six seconds. Since we had two blocks, we needed 90 trials. To select these 90 trials we relied on Bowden and Jung-Beeman's study data. That data included a list of 144 CRA trials ordered by the percentages of participants who were able to solve a given CRA trial in 15 seconds or less, where the start of the list included the easiest CRA task (majority of participants were able to solve it), and the bottom the hardest (few participants were able to solve it). For instance, the CRA trial cream/skate/water was solved by $96 \%$ of participants in that time limit or less, the next trial in the list loser/throat/spot was solve by $86 \%$, and so on. From this list, we selected the top 90 words (from the authors' perspective, this gave us a solvability range between $96 \%$ at the easiest trial, and $18 \%$ at the hardest trial. To control for difficulty (i.e. to ensure participants did not receive harder or easier questions), we divided the 90 trials into three sets of 30 , so that set 1 contained the 30 easiest questions, set 2 had medium difficulty and set 3 had the 30 hardest questions. To populate block 1, trial 1 was taken from set 1 , trial 2 from set 2 , trial 3 from set 3 , and this procedure repeated 
sequentially for trials 4 (taken from set 1), 5 (taken from set 2), 6 (taken from set 3), and so on. The order of presentation was randomized so that difficulty order varied within each block and fixed within that order so that all participants were presented with the same order of questions, ensuring that performance differences were not attributable to variance in difficulty.

Non-creative control task

The non-creative task was a convergent-thinking counting task. To avoid bias, we used the same underlying materials in the control task as for the CRA task, where the control also involved word triplets, but instead required finding the difference between the total number of consonants and vowels across all three stimuli words. Take for example the triplet fruit/ line/ drunk. There are nine consonants and five vowels. Thus, the correct response for the difference between consonants and vowels is four. For simplicity, participants were told to count $y$ as a consonant regardless of location within the word.

\subsubsection{EEG equipment}

\section{Data acquisition}

The experimental (CRA) and control (non-creative) task stimuli were presented to participants on a monitor, which was located in the Faraday cage as it was also used for EEG data collection. Raw EEG data was collected using a 128-channel HydroCel Geodesic Sensor Net (HCGSN), a GES 250 amplifier to sample, filter and digitize data, and Net Station v. 4.3.1 for recording (see Figure 5.1 for set up overview). Impedance was set at $\leq 50 \mathrm{k} \Omega$, which uses a small voltage metre to ensure all EEG electrodes are in good contact with the scalp to produce clean EEG signals. 


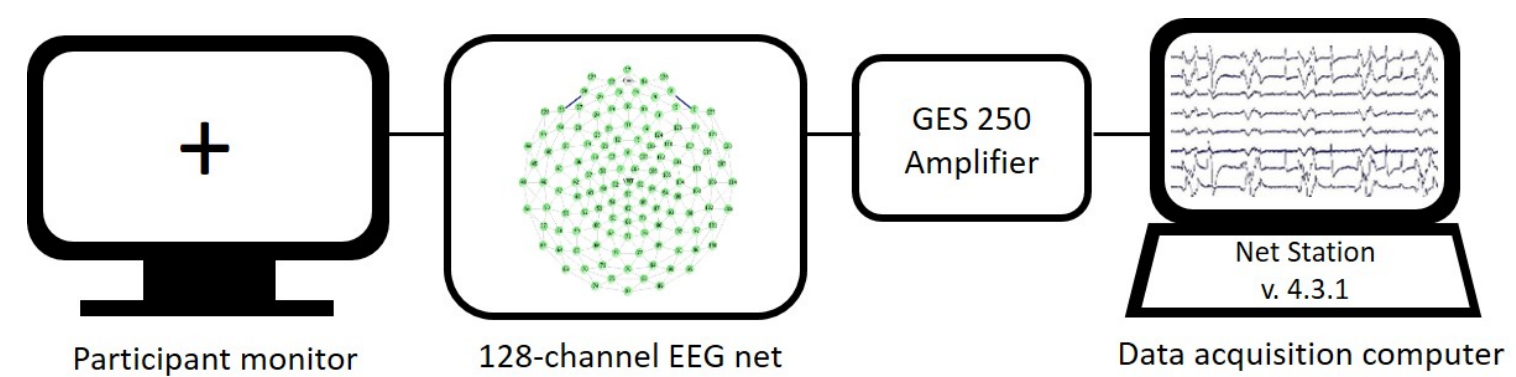

Figure 5.1: Experimental set up. Participants were presented with a fixation cross to focus upon for the duration of the session while listening to auditory clicks.

\section{Paired click paradigm}

To measure attention, we used the standard paired-click paradigm for early sensory gating. Participants used sanitized, generic brand earbuds to listen to the clicks. Clicks 1 (C1) and 2 (C2) were presented at 500ms intervals. Each click lasted 1ms. Click pairs were presented at pseudo-randomized inter-pair intervals of 6 , 8, or 10 seconds, which reduced the possibility of the participant anticipating the timing of the next click pair, thereby controlling for expectancy effects. A total of 100 click pairs were presented, lasting approximately thirteen minutes (see Figure 5.2).

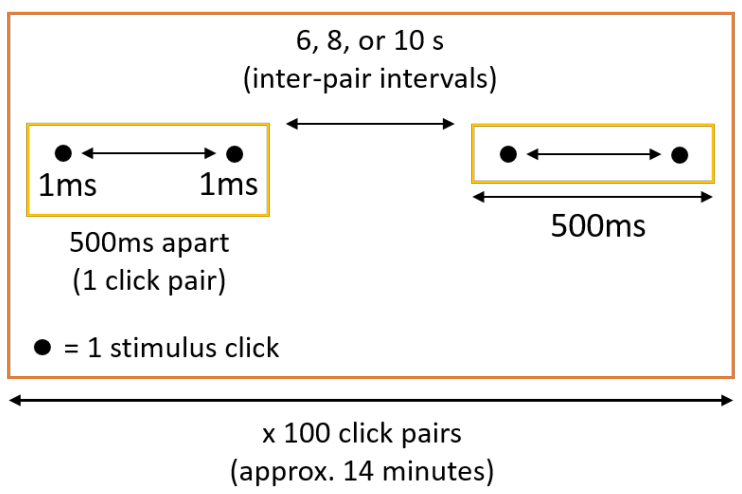

Figure 5.2: Layout of the paired-click paradigm in the present study. 


\subsection{Procedure}

Experimental sessions were conducted individually and lasted between 100-120 minutes (the variability in time is due to time needed to adjust the EEG net and electrodes). Prior to each session, written consent was obtained from participants in accordance with Carleton University ethics.

Each experimental session was broken up into two sub-phases. In the first phase, we assessed participants' divergent, convergent, and non-creative thinking abilities, as per the following. Participants first completed the ATTA divergent thinking task, a paper and pencil task that takes approximately 10-13 minutes to administer. Next they were seated in front of a monitor where the stimuli for the CRA and control tasks were presented. Prior to starting the session, participants were notified they would be working through two sets of tasks, called a "word task" (CRA) and a "number task" (control). The experimenter delivered instructions verbally (see Appendix C), which were also presented on screen for participants to follow along. They were given three practice trials for each task to ensure they understood the task during which they were encouraged to ask questions and clarify the task if needed. In the experimental session, a single trial began with the word triplet appearing on the screen (Figure 5.3 A). When the participant had an answer in mind, s/he pressed a button on the button box (Figure $5.3 \mathrm{~B}$ ), which brought up a screen prompting them to say the answer aloud (Figure $5.3 \mathrm{C}$ ); the answers were recorded by the experimenter (Figure $5.3 \mathrm{D}$ ). Once the participant answered, they pressed the button to proceed to the next trial (Figure $5.3 \mathrm{~F}$ ). After 15 seconds, if the participant had not answered, the prompt

screen was replaced by a time-out screen (Figure $5.3 \mathrm{E}$ ), which again prompted the participant to press to continue. 


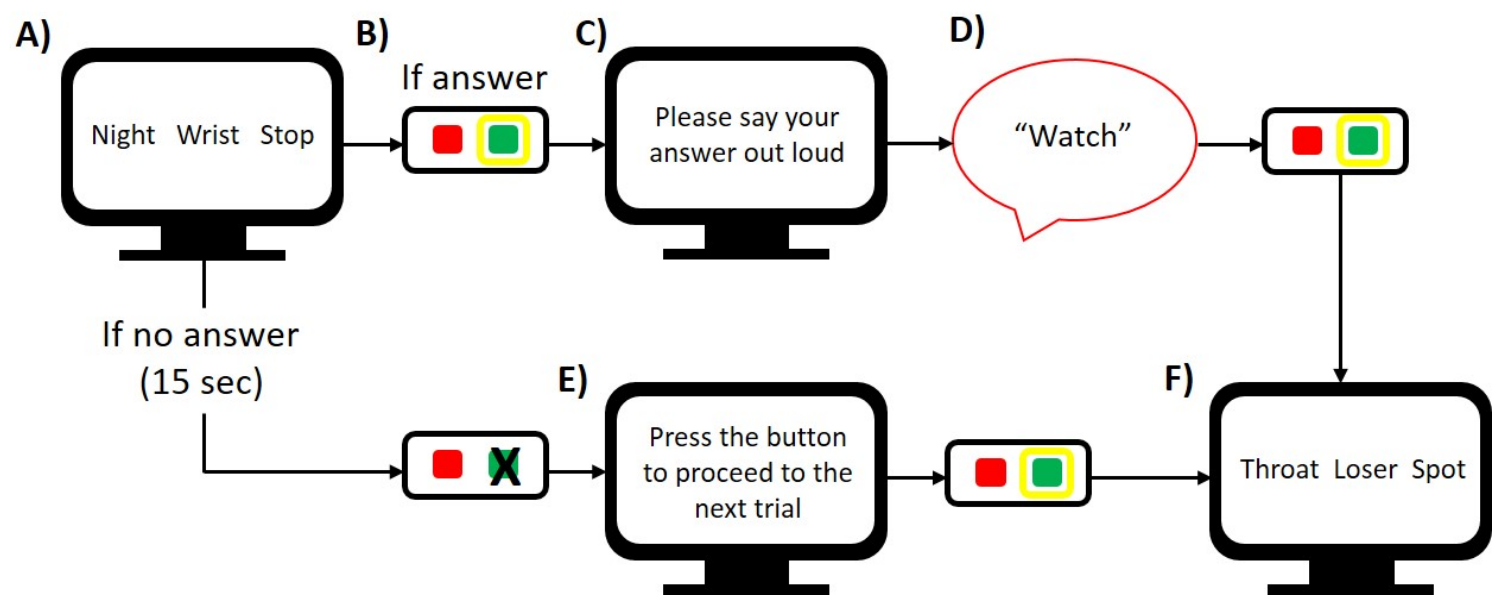

Figure 5.3: Step-by-step illustration of the experimental process for the CRA and the control tasks.

This procedure was chosen to maximize accuracy in recording the creative process so that participants were not exposed to solution hints at any point. We also chose the 15-second limit so that answers were based on the ability to create loose associations, as opposed to a trial-and-error process, and to maximize the number of trials.

A given session involved a total of four blocks, each lasting exactly five minutes, with a self-timed break between blocks. Each block consisted either of CRA trials or control task trials; there were two blocks of each (CRA, control). The order of the blocks was the same for each participant, so that each session began with a CRA block, followed by a control block, a second CRA block, and a final control block. We chose this order to prevent training effects, so that the stimuli words from the CRA block could be recycled in a randomized order for the following control task block. Because the counting task words were always the same as the CRA words from the preceding block, participants were not exposed to novel words that could trigger associations for the CRA task.

Once the three assessment tasks were completed, we proceeded with EEG data 
collection. This phase included the following sub-phases:

\section{Preparation}

2. EEG data collection data cleaning and pre-processing

\subsubsection{Preparation}

The participant's head circumference was measured to select the correct net size (L, $\mathrm{M}, \mathrm{S})$ and the EEG net was prepared as per the HCGSN manufacturer instructions. The net was soaked in an electrolyte solution consisting of distilled water, baby shampoo, and potassium chloride for 5-10 minutes. While the net was soaking, participants were asked to wash their hair, which improves the quality of the data collected by helping to rid the scalp of any salts that may have accumulated over the day, as well as keeps the net clean. They were also asked to blow dry their hair so that it $95 \%$ dry. We then fit EEG over the participants head. To ensure consistent placement, we followed the standard procedure, as follows. The central reference point when placing the net over the scalp is labelled the $\mathrm{Cz}$ and located at the vertex. The vertex is the top of the scalp where the midpoint of the nasion (where the bridge of the nose meets the forehead) and the inion (the small bump at the back of the skull) intersect with the midpoint of the pre-auricular points (the area where the jaw meets the skull). After aligning the $\mathrm{Cz}$ electrode with the marked vertex point, the net is slipped over

the head, ensuring that the ears and eyes are within the corresponding cut out holes. Once securely in place, the net is connected to the amplifier that collects and runs the signals to the data acquisition computer. The final phase before data collection corresponded to impedance measurements. 


\subsubsection{EEG data collection}

Following the preparation stage, participants were seated comfortably in front of the monitor and given earbuds to secure in their ears. They were instructed to refrain from making sudden movements and talking out loud in order to avoid interfering with the data collection given the sensitivity of the EEG to external noise and movement. They were also notified that this part of the experiment would last thirteen minutes, and that if at any time they felt discomfort, they were free to notify the experimenter and stop the study. The paired- click paradigm then began, which, as described above, involved a series of two clicks played in close succession at regular intervals.

\subsection{EEG data cleaning and pre-processing}

Twenty-five participants were included $(F / M=12 / 13)$. Of the original set of 36 , four participant files produced unusable data: two individuals had hair extensions and the electrodes did not sit well enough, and two individuals had little to no hair which caused bridging effects (often caused by electrolyte solution spreading between electrodes, resulting in inaccurate signal recording). For three other participants, data did not record properly due to the display monitor malfunctioning; two more participants were excluded on the basis that they did not complete any of the three tasks correctly.

As is standard, the raw data files were corrected for timing accuracy using Net Station waveform tools. We calculated a 60s timing offset between on-screen stimulus presentation and recording. The raw files were then exported to EEGLAB for data cleaning.

Data cleaning and processing was done using EEGLAB, a MATLAB toolbox. 
Darbelia, an EEGLAB plugin, was used to process the files serially. The HCGSN uses electrode locations $68,73,88,94$ as connectors to the amplifier; these may be called "sacrifice channels" and are removed in EEGLAB. A notch-filter was applied between the standard 58-62 $\mathrm{Hz}$ range to remove electrical noise. Each file was manually inspected for noisy channels, then bandpass filtered between 1 and $70 \mathrm{~Hz}$ (Zabelina et al., 2015). Average re-referencing was computed post-filtering as well as after epoch extraction. Epochs were segmented from -200 ms to $800 \mathrm{~ms}$ post-stimulus onset and baseline corrected at $-190 \mathrm{~ms}$ to $0 \mathrm{~ms}$. This larger epoch was chosen to produce a clean data set, as a smaller epoch length resulted in a very uneven baseline specific to the second click. Ocular and muscular artifacts were removed, as well as trials with values exceeding $75 \Omega \mathrm{V}$ (Lijffijt et al., 2009).

\subsubsection{Component filtering and extraction}

Because differential cognitive processing occurs within different frequencies, existing literature suggests various filter settings for optimal data extraction specific to each component. Due to the noted variability and small amplitude values of the P50 component, Jerger et al. (1992) note that the P50 'rides' on the slope of the N100, suggesting that the amplitude of the P50 is easily attenuated by the N100. According to Lijffijt et al. (2009) this can be countered using an appropriate filter setting, specifically that "a filter setting lower than $10-\mathrm{Hz}$ would not sufficiently suppress the N100 and could subsequently reduce the amplitude of the P50." Following this advice, we applied component-specific filters to optimize scoring of the P50, N100 and P200 latencies: A $10 \mathrm{~Hz}$ high-pass filter for the P50 and a $20 \mathrm{~Hz}$ low-pass filter for the N100 and P200 components (Jerger et al., 1992).

After cleaning and processing ERP data for P50 components, five participant files 
were discarded because waveforms deviated from a "typical" formation (see Figure 5.4). Twenty-two files were included in the final P50 analysis $(F / M=10 / 12)$.

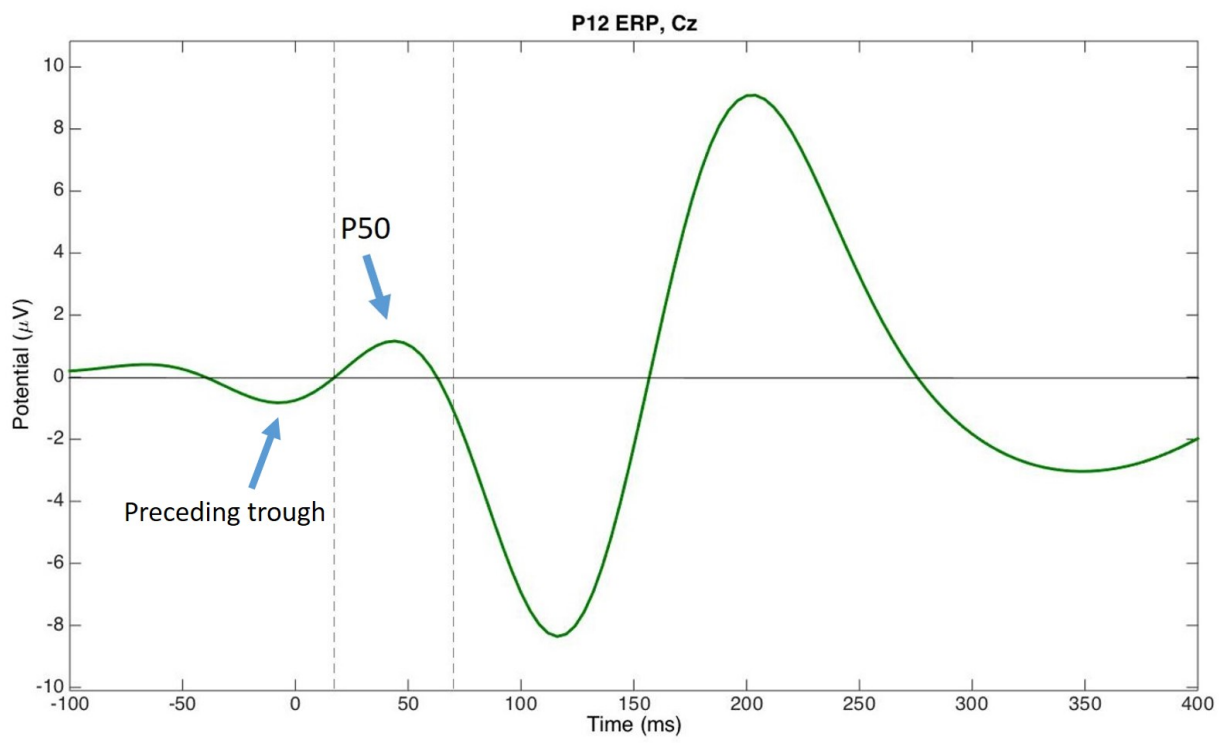

Figure 5.4: A standard P50 component. Peak occurs within the $30-80$ ms window, and is immediately preceded by a trough. Data files were visually inspected on an individual basis and were excluded if the P50 peak deviated from either of these conditions.

Because a different filter was applied for the N100 and P200 components, we extracted these separately from the P50 but using a similar approach. In both cases, the trough had to be clearly isolated from the preceding peak or trough. Twenty-three files were included in this analysis $(F / M=11 / 12)$. 


\section{Chapter 6 Results}

\subsection{Statistical analysis}

SPSS and EEGLAB were used for statistical analysis. Relationships between all independent and dependent variables were inspected visually for outliers; none were observed. Pearson correlation coefficients were computed to investigate relationships between P50 components and performance on assessment tasks. We also conducted correlational analyses between assessment tasks and the N100 and P200 components to assess the specificity of creativity relationships to the P50. Sensory gating was indexed by subtracting the amplitude value of the second click from the first click (C1 - C2) (Lijffijt et al., 2009; Rentzsch, Jockers-Scherübl, Boutros, \& Gallinat, 2008). Recall from the methods section that the sample size for the P50 component $(n=22)$ differed from the $\mathrm{N} 100$ and P200 component $(n=23)$ during extraction. Descriptive data presented in the table below is based on the P50 group since this was our focus component; subsequent analysis concerning the N100 and P200 use the corresponding data sets.

\subsection{Descriptive data: Divergent and convergent thinking test scores}

Table 6.1 displays descriptive statistics for performance on divergent and convergent

thinking tasks. To assess divergent thinking, we considered scores for fluency, orig- 
inality, as well as fluency plus two times originality Zabelina et al. (2015). Given the recent focus on ideational creativity (i.e. complete test scores), we also used the overall ATTA score (Runco \& Mraz, 1992).

Table 6.1: Performance scores from assessments of divergent and convergent thinking tests of creativity and non-creativity (control task).

\begin{tabular}{llll}
\hline Variable & $N$ & $M$ & $S D$ \\
\hline Divergent thinking & & & \\
Fluency & 22 & 11.86 & 3.57 \\
Originality & 22 & 3.91 & 2.31 \\
F+2 $(\mathrm{O})^{1}$ & 22 & 19.68 & 7.15 \\
Total scores & 22 & 62.82 & 16.97 \\
Convergent thinking & & & \\
CRA & 22 & 11.18 & 5.70 \\
Control & 22 & 24.73 & 15.17 \\
\hline
\end{tabular}

Note $1 . \mathrm{F}+2(\mathrm{O})=$ Fluency

score plus two times origi-

nality score.

\subsection{EEG results}

\subsubsection{Manipulation check}

As the first step post data filtering, we performed a manipulation check to determine that the two tone paradigm had the intended effect. P50 peak latencies were extracted from the standard 30-80 ms range (Figure 6.1 A), N100 latencies were extracted using the value of the minimum most point within the $80-150 \mathrm{~ms}$ window (Figure 6.1 B), and P200 latencies were extracted using values of the maximum peak within a 150-250 ms time window (Figure 6.1 C). Significance $(p \leq .05)$ was computed with EEGLAB statistics and was observed across all $\mathrm{C} 1$ and $\mathrm{C} 2$ comparisons. In Figure 6.1 D, significance between neural responses is represented by the black bars on the 
$x$ axis. As expected, averaged waveform amplitudes for all three components (P50, N100, P200) showed that neural responses were significantly higher at C1 than at C2 (where $\mathrm{C} 1=$ amplitude of ERP for the first click and $\mathrm{C} 2=$ amplitude of ERP for the second click).

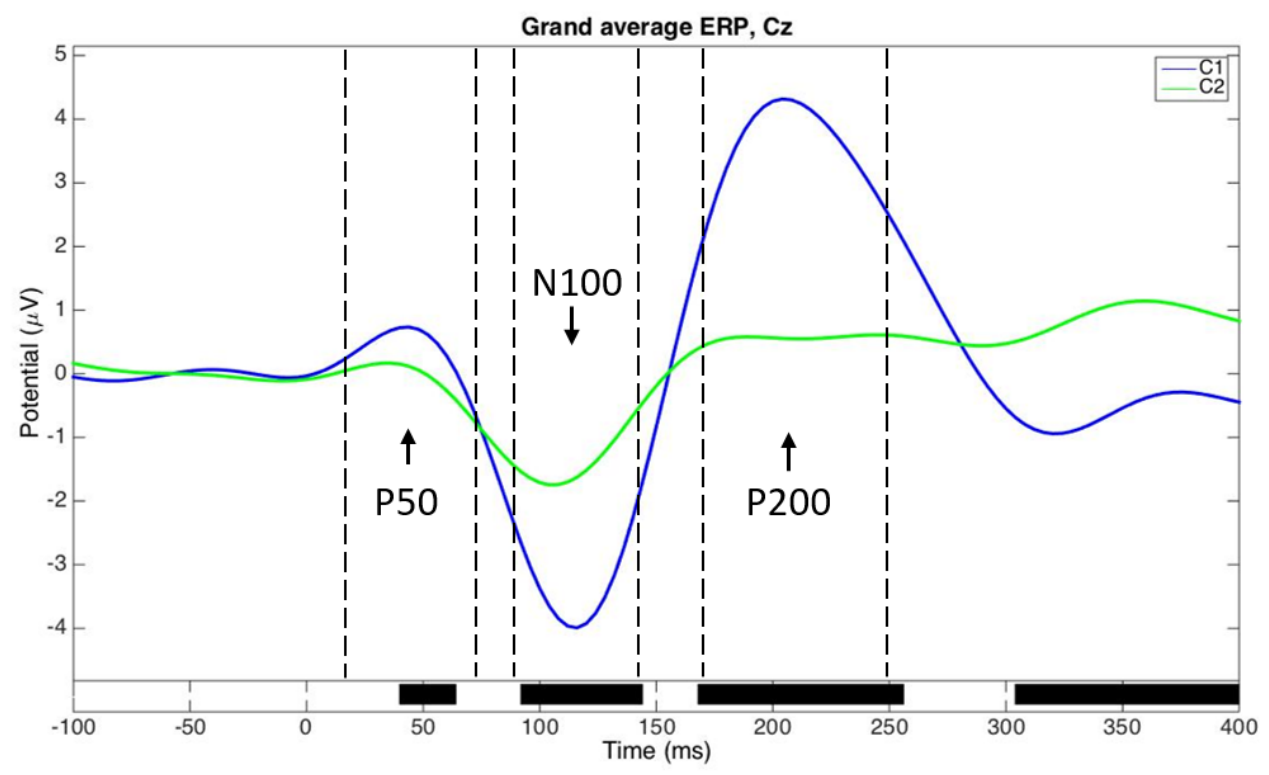

Figure 6.1: As indicated by the dashed lines, (A) P50 amplitudes were extracted between 30-80ms; (B) N100 between 80-150ms, (C) P200 between 150-250ms. (D) Black bars beneath components show significance in click responses across participants for each component $(p \leq 05)$. 


\subsubsection{P50, N100 and P200 amplitude and suppression values}

ERP amplitude values for P50, N100 and P200 are presented in Table 6.2.

Table 6.2: ERP values for Click 1 (C1), Click 2 (C2), and Click 1 Click 2 (C1-C2).

\begin{tabular}{llll}
\hline ERP component & $N$ & $M$ & $S D$ \\
\hline P50 & & & \\
C1 & 22 & 0.64 & 0.69 \\
C2 & 22 & 0.46 & 0.57 \\
C1-C2 & 22 & 0.17 & 0.57 \\
N100 & & & \\
C1 & 23 & -5.12 & 2.35 \\
C2 & 23 & -3.51 & 1.70 \\
C1-C2 & 23 & -1.61 & 0.93 \\
P200 & & & \\
C1 & 23 & 5.30 & 2.58 \\
C2 & 23 & 3.10 & 1.39 \\
C1-C2 & 23 & 2.20 & 1.44 \\
\hline
\end{tabular}

\subsection{Correlational results}

\subsubsection{P50 and divergent thinking}

There was a strong negative correlation between fluency scores and sensory gating with a medium effect size, $r(22)=-.46, p=.03$. (Figure 6.2), indicating that individuals scoring highly on the fluency aspect of creativity were associated with more defocused attention. 


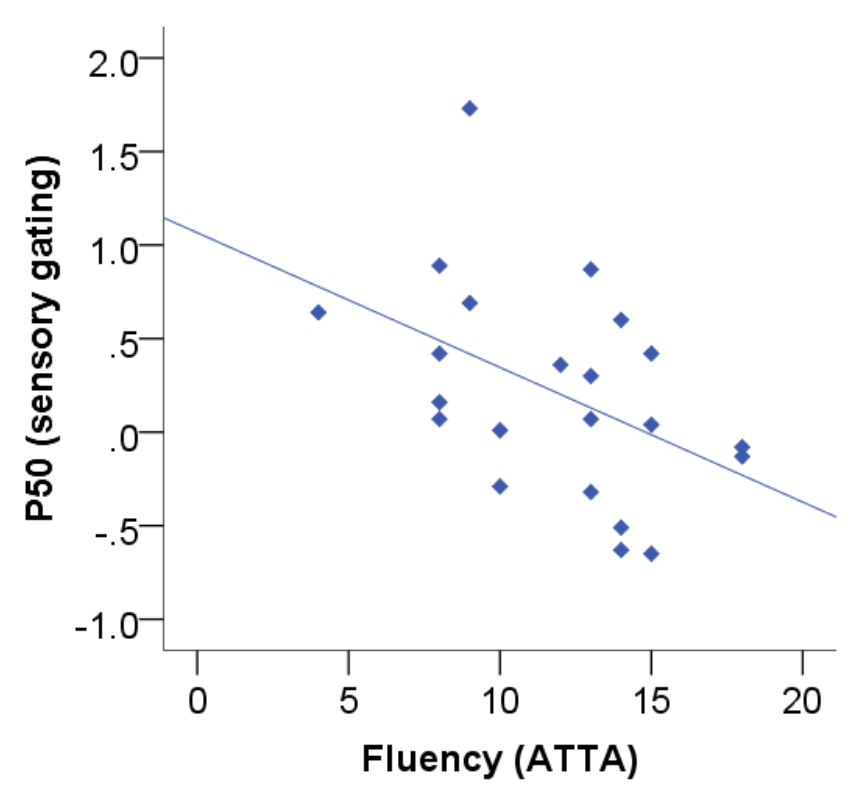

Figure 6.2: A negative correlation observed between Fluency scores on the divergent thinking test of creativity, and sensory gating. Divergent thinking was associated with a more defocused attention style.

Associations between sensory gating and remaining divergent thinking scores (overall ATTA, fluency plus originality, and originality) were non-significant: a) overall ATTA, $r(22)=.10, p=.66$, b) fluency plus two times originality, $r(22)=-.14, p=$ .53 , and c) originality $r(22)=-.06, p=.79$.

\subsubsection{P50 and convergent thinking}

A significant positive association was observed between sensory gating and both the creative and non-creative convergent thinking tasks. Enhanced performance on the CRA correlated positively with defocused (leakier) attention, $r(22)=.73, p<.01$, with a large effect size, (see Figure 6.3), as did performance on the non-creative convergent task, $r(22)=.53, p=.01$ with a medium effect size (see Figure 6.4). 


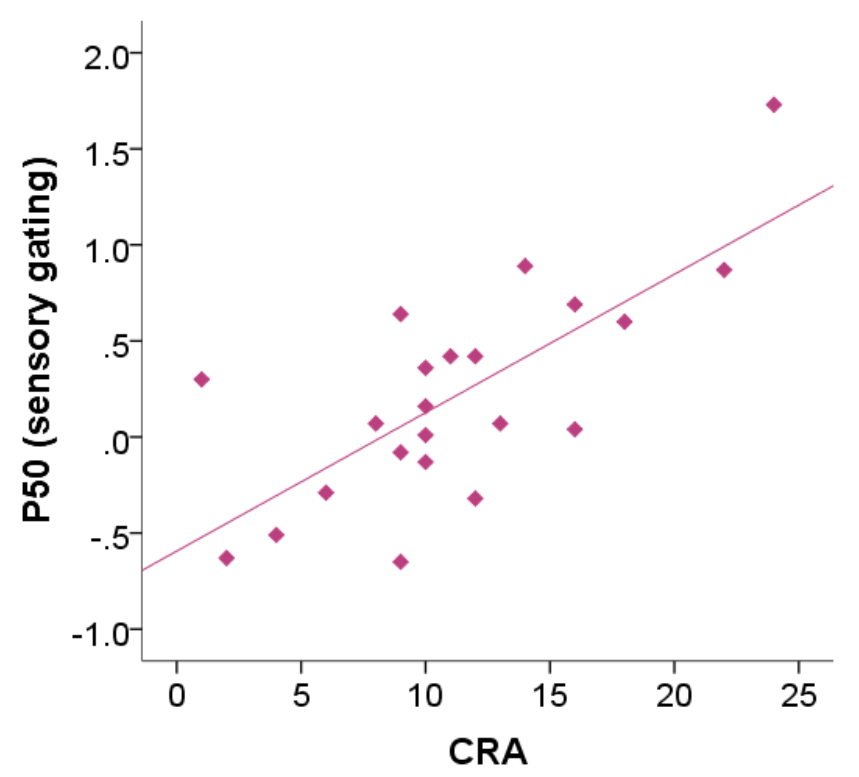

Figure 6.3: A positive association between CRA scores and sensory gating, indicating focused attention.

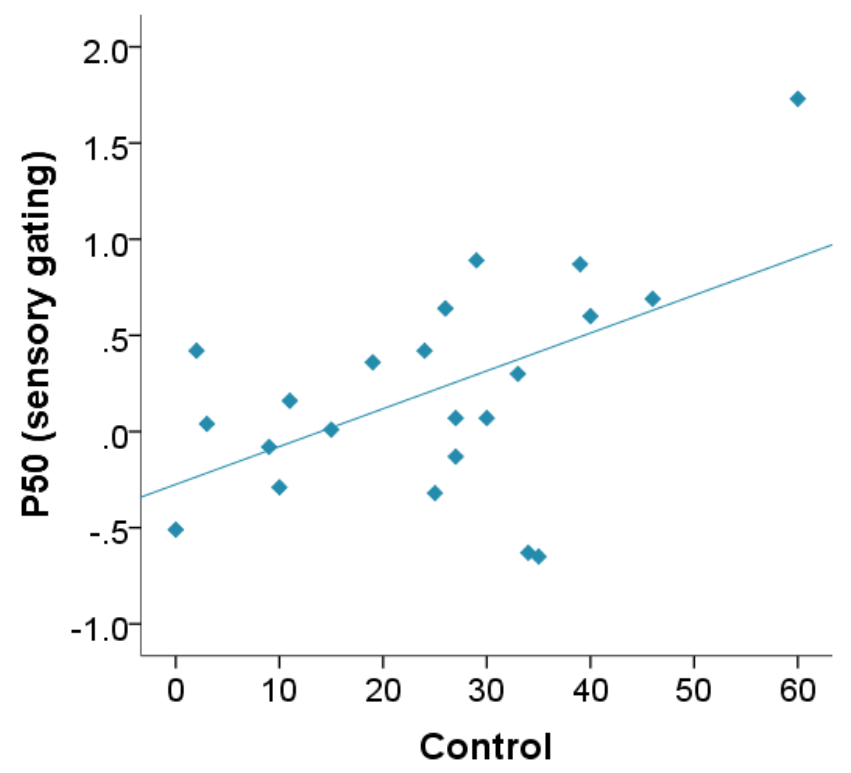

Figure 6.4: A positive correlation between the convergent, non-creative task and sensory gating. 


\subsubsection{N100, P200 and performance scores}

We also ran a correlational analysis between the other components of interest (N100 and P200) and performance scores to determine whether sensory gating effects on creativity involved these later components. As displayed in Table 6.3, none of the relationships were significant, indicating that the above mentioned relationships were specific to the P50 component.

Table 6.3: Pearsons $r$ and $p$ values reported for correlations conducted between N100 and P200 component and assessment tasks.

\begin{tabular}{lllll}
\hline \multirow{2}{*}{ Task } & \multicolumn{2}{c}{ N100 } & \multicolumn{2}{c}{ P200 } \\
\hline Fluency & $p$ & $r$ & $p$ & $r$ \\
Originality & .62 & .11 & .13 & -.33 \\
F+2(O) & .93 & -.02 & .53 & -.14 \\
Overall ATTA & .62 & .11 & .95 & -.02 \\
CRA & .12 & -.33 & .32 & -.22 \\
Control & .30 & -.23 & .29 & .23 \\
\hline
\end{tabular}

\subsubsection{Correlations among tasks}

We were interested in correlations between performance on the divergent, convergent and non-creative thinking tasks. A positive relationship was found between the CRA and overall ATTA scores, $r(25)=.43, p=.03$ (see Figure 6.5$)$, showing that ideational creativity performance was similar in individuals across both divergent and convergent styles of creativity.

Additionally, a positive relationship was found between the CRA and control task, $r(25)=.52, p<.01$ (see Figure 6.6), indicating that within-subject performance was similar on both convergent thinking tasks. Relationships did not appear significant between the CRA and fluency scores $r(25)=-.10, p=.62$, nor between CRA and 


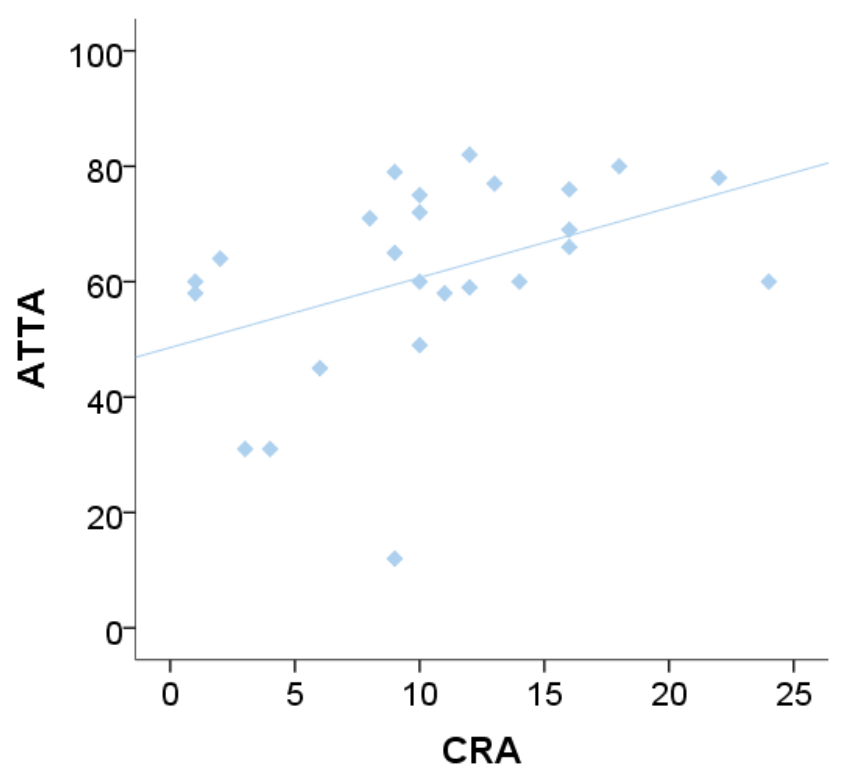

Figure 6.5: A positive correlation between the convergent, non-creative task and sensory gating.

originality $r(25)=.28, p=.17$.

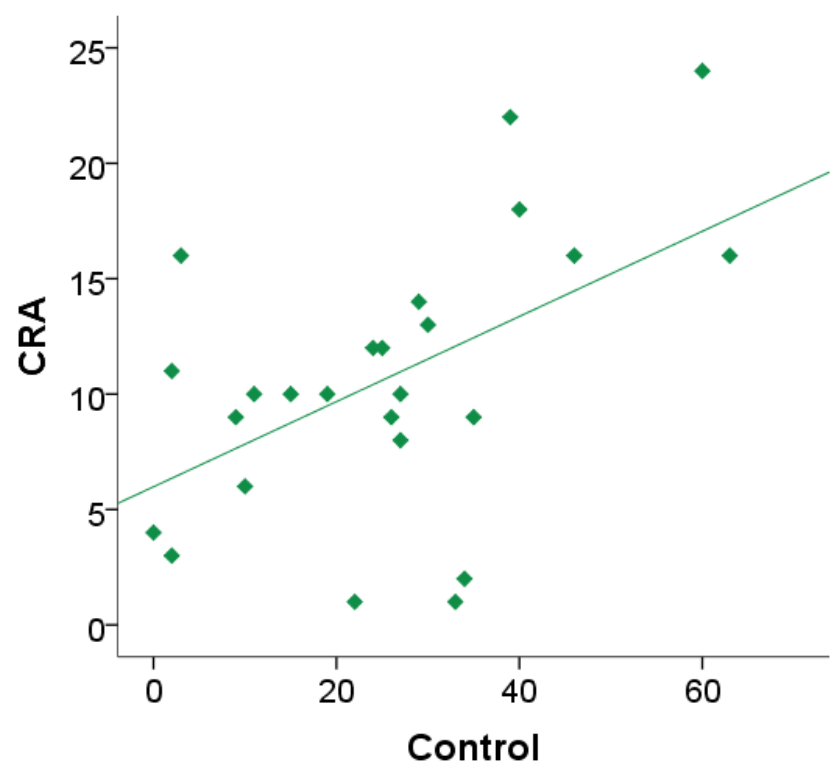

Figure 6.6: Creative and non-creative convergent thinking tasks show a strong positive association. 
The non-creative task and overall ATTA scores did not correlate significantly, $r(25)=.36, p=.08$, although visual inspection shows a trend towards a positive relationship (Figure 6.7).

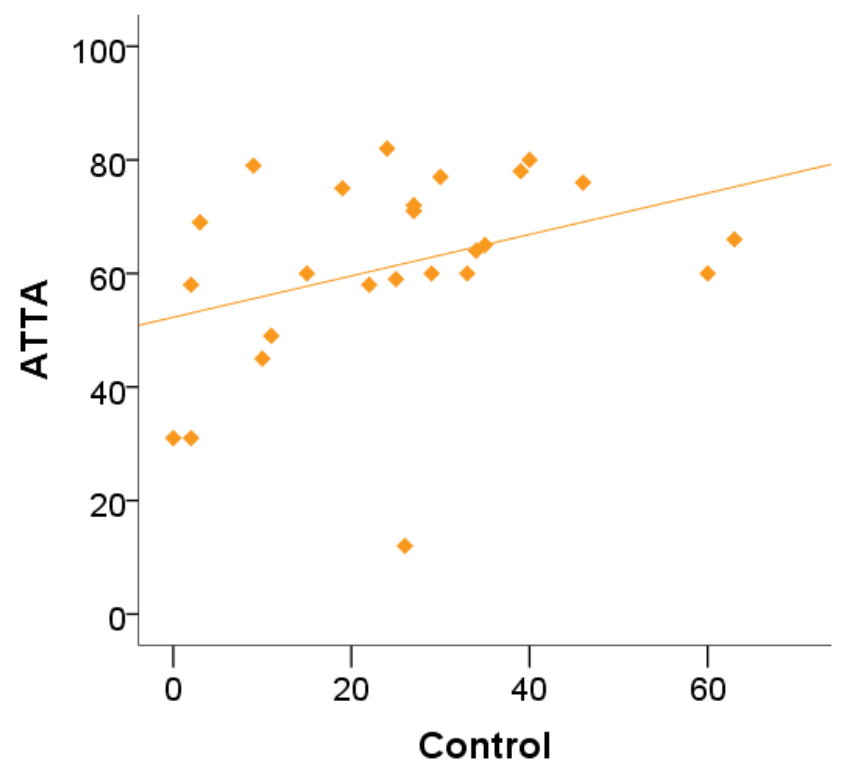

Figure 6.7: The relationship between the ATTA and control task performance appear to be trending towards a positive correlation. 


\section{Chapter 7 Discussion and Conclusion}

\subsection{Interpretation of results}

In order to investigate the impact of attention on creativity, attention styles were indexed by the P50 sensory gating component that measures the strength of an individual's ability to filter irrelevant stimuli. We employed divergent, convergent and non-creative thinking tasks and assessed relationships between performance on these tasks and sensory gating. Based on prior findings that divergent thinking has been associated with defocused attention (i.e. Carson et al., 2003; Fiore et al., 2001) and convergent thinking with focused attention (i.e. Martindale, 1999; Necka, 1999), we hypothesized that sensory gating would be negatively associated with ATTA scores (hypothesis 1) and positively associated with the CRA (hypothesis 2). Our correlational analysis revealed some interesting results, as does a methodological evaluation of our ERP data.

\subsubsection{Creative performance and sensory gating}

First, our hypothesis proved partially correct in that the ATTA, the divergent measure of creativity, was negatively associated with sensory gating. We found a negative relationship between fluency and decreased sensory gating, indicating that individuals who scored higher on fluency in a divergent thinking task show more defocused 
attention. This finding differed from that of (Zabelina et al., 2015), who found no relationship between fluency and sensory gating, but found that originality correlated positively with early sensory gating. We suggest two possible reasons for this discrepancy in results, which will be elaborated upon in the following sections: 1) according to the existing literature, originality may be one of the less reliable measures of divergent thinking, and 2) we used very precise filters in extracting ERP data, which the previous authors did not, and it is likely this skewed their sensory gating values.

Secondly, our hypothesis concerning convergent thinking was proven correct by a positive relationship between the CRA and sensory gating. Thus we conclude that a more focused attention style mediates the generation of creative responses that require single, correct answers. This was further strengthened by the finding that focused attention also correlates positively with non-creative, convergent thinking performance, indicating that attentional style can be specific to cognitive processing in both creative and non-creative domains.

In terms of sensory gating, these relationships demonstrate that poor inhibitory control (represented by the inability to filter task-irrelevant information at an early stage) is advantageous for divergent thinking tasks, but is disadvantageous for convergent thinking tasks. Such a notion is supported by clinical studies involving ADHD and non-ADHD individuals, since ADHD is directly characterized by poor inhibitory control that interferes with working memory (i.e. Barkley, 1997). In White and Shah (2006)'s study, individuals with ADHD (defocused attention) performed better on a divergent thinking task, but worse on the RAT compared to their non-ADHD (focused attention) counterparts.

The remaining aspects of the ATTA we evaluated (originality, fluency plus two 
times originality, overall ATTA), did not present significant relationships with early sensory gating. These aspects all involve a measure of originality. As such, our finding is not surprising in light of previous research that has evaluated the reliability of the originality score in divergent thinking tests. For example, Hocevar (1979) reported that originality was only "spuriously reliable and valid" (although test limitations were noted in his work), and Runco and Albert (1985) further suggested that originality scores may only be reliable in gifted populations. These studies provide one explanation for the relationship we found between fluency and attention, but not between originality-related scores and attention. As mentioned earlier, the study by (Zabelina et al., 2015) did find that individuals who performed better on the originality portion of the ATTA showed more focused attention. However, the aforementioned studies raise some questions about the reliability of this relationship. That this relationship (divergent thinking mediated by focused attention) is not typ-

ically witnessed in creativity literature further alludes to the questionable nature of this finding. That being said, we also speculate this discrepancy may be due to an oversight in correctly filtering data specific to the P50, discussed shortly.

\subsubsection{Non-creative performance and sensory gating}

We also observed a positive correlation between the non-creative, convergent thinking task and sensory gating, suggesting that high-performing individuals on this task maintained more focused attention. This finding is particularly interesting as we were not speculating strong associations between the non-creative task and attention styles; however, it is in line with the CRA-related result and both are convergent thinking tasks. This further suggests that focused attention is a mediator of convergent thinking as an overall cognitive process, and not only specific to convergent thinking in 
creative situations. In particular, one cognitive process that both the CRA and the counting task invoke is working memory. Generation of associative concepts on the RAT (and likewise CRA) invokes a great deal of working memory to retrieve lexical and semantic concepts; according to Fiore et al. (2001), inhibitory control is needed to keep the individual on task until a solution is reached. After retrieving from memory, the individual further needs to assess the element relative to the stimuli words and choose to either continue searching or confirm the solution. Similarly, counting tasks in general and our task in particular require a strong working memory capacity. In our task, the individual counted consonants, stored that number in working memory, counted vowels, retrieved the consonant number, performed a routine subtraction and confirmed the solution. Thus we propose that focused attention mediates convergent thinking in both creative and non-creative domains.

\subsubsection{Correlations among tasks}

When we assessed relationships between tasks we found a strong positive correlation between CRA and control task scores, which is consistent with the above-described results for the corresponding attentional relationships. In contrast to the attentionrelated results, however, we also observed a positive correlation between overall ATTA and CRA scores as well as between the control task and ATTA. One possible explanation for this discrepancy could be the influence of other cognitive processes driving performance. We only investigated attention, but as we have seen, creativity is influenced by a variety of factors. Working memory, for example, was discussed earlier in terms of the CRA and has also been attributed to influencing divergent thinking tasks (i.e. De Dreu, Nijstad, Baas, Wolsink, \& Roskes, 2012). 


\subsubsection{EEG results: A methodological evaluation}

Filtering of EEG data is a key factor that contributes to cleanliness and accuracy of data. The P50 component in particular is highly sensitive to this since its small amplitude and early latency can make it difficult to detect. Furthermore, its waveform borders on that of the N100 component, which is much larger and more robust, and can easily diminish the amplitude of the P50 (Jerger et al., 1992). Unfortunately, data cleaning procedures in EEG generally, and for the P50 specifically, lack standardization in the cognitive neuroscience field. This can lead to unecessarily discarded data if component waveforms cannot be detected, or incorrect amplitude values, if neighbouring components interfere. In the present work, we take a step forward in this direction by demonstrating the importance of correctly preparing data to work with the P50. We prevented carry-over effects by using a very reliable method that ensured an isolated P50 component, specifically filtering between 10-50 Hz. This also allowed us to retain several data files that would have been discarded had a more general filter been used, since a clear waveform could not be detected. We mentioned earlier that Zabelina et al. (2015)'s findings may have differed due to their P50 extrac-

tion methods. These authors used a much more general filter $(1-70 \mathrm{~Hz})$ that would not have controlled for N100 effects, and one possibility is that their P50 component values were skewed by the impeding N100 values.

\subsection{Concluding remarks}

The aim of this research was to examine how individual differences in attentional styles influence performance on divergent and convergent thinking tests of creativity. We were successful in demonstrating that (1) defocused attention best mediates divergent 
thinking tasks, and (2) focused attention best mediates convergent thinking tasks. This work contributes to the field by highlighting the role of attention in standard lab-based creative tasks and in particular that attention interacts with the type of task to influence outcomes. For the first time, we show relationships between early attention and cognitive processing styles.

A secondary goal of this research was to examine the use of ERP data in the field of creativity, with a focus on the P50 component. The P50 is the least studied component, not for lack of importance, but for the challenges related to its measurement (S. Luck \& Kappenman, 2011), and so work in this area is much needed. Our work adds to the neuroscience field by demonstrating the utility of this component for studying early attention. To address the challenges related to the measurement of the P50, we used a combination of general methods (i.e. ocular and muscular rejection), and component-specific methods (i.e. time windows, $10 \mathrm{~Hz}$ high-pass filter), in particular the importance of pre-processing specific to the component. Future work in the realm of the P50 component (with or without creativity applications) would benefit from the application of such standardized methods.

\subsubsection{Limitations}

Limitations in this project were both EEG and task related. The main limitation with EEG recordings is susceptibility to noise, which we were unable to control. This forced us to discard some data, consequently reducing our sample size. Additionally, the P50 paradigm is a lengthy one, and while we followed the standard protocol, it is possible that some participants experienced difficulty staring at the screen without movement, resulting in discarded trials.

A task-related limitation pertained to the tasks we used. First, as far as the CRA, 
even though we provided instructions and practice trials, several participants did not comprehend the idea of creating "compound phrases" (this data was discarded). In general, the tasks and measures used in our work are standard and established in creativity research in general (e.g., Torrance test) and EEG creativity work in particular (e.g., CRA). However, we are not claiming that these tasks are representative of ones involved in real-world creativity, such as that required for scientific and artistic endeavours. Thus, it is an open question as to how our findings apply to these real-world settings.

\subsubsection{Future work}

An interesting extension to this work would be to have participants complete the CRA whilst undergoing the EEG P50 paradigm, in order to get closer to the neural underpinnings of convergent creative thought during task completion. Future work may also to investigate working memory in conjunction with these tasks, since it has been shown to influence divergent thinking, and clearly played a role in the convergent thinking tasks but has not been examined in the context of all three tasks. Yet another avenue of future work could pertain to exploring the role of attention in more complex creative endeavours than ones used here. Doing so, however, would require shifting paradigms from EEG, given the methodological restrictions of EEG. Finally, it would also be interesting to explore if interventions related to attention could be used to increase creativity. The incorporation of creative thinking interventions is currently a hot topic in industrial and organizational settings (see Handbook of Organizational Creativity). Thus, it would be interesting to see if measuring and accommodating individual attentional differences could influence cognitive creative process skills and task motivation and perhaps lead to expertise in task-dependent situations. For 
instance, understanding an individual's attention style in the workplace or classroom can hint at the kind of creative task s/he would more likely excel at. 


\section{Glossary}

Action potential The electrical activity that occurs when a cell is excited and the membrane potential has reached threshold.

Artifacts (EEG recording) Voltage spikes in the data that are due to non-cognitive processing, such as eye blinks or background noise. Artifacts are removed in the early stages of pre-processing EEG data.

Attention A set of processes that control the flow of information through the nervous system.

Attenuation model A bottleneck model of attention proposed by Treisman in which she suggests that unattended information is attenuated, but not completely discarded as in the filter model.

Axon terminal The endpoints of branches of an axon through which chemical and electrical messages can be sent from presynaptic neurons.

Band-pass filter A filter that allows frequencies within a set range to pass, and rejects frequencies outside that range.

Bottleneck theories Attention theories that view attention as a limited resource and suggest the presence of cognitive mechanisms that filter information as it enters the brain.

Complexity The ability to mentally retain interrelated factors of the conceptual structure of the problem and to resolve these simultaneously. 
Component (ERP) A set of voltage changes that are consistent with a single neural generator site and that systemically vary in amplitude across conditions, time, individuals, and so forth. These voltage changes occur in response to specific events or stimuli and appear as waveforms in the EEG recording, which are then associated with specific forms of cognitive processing.

Compound Remote Associates (CRA) A subset of the Remote Associates Task, adapted for ease with EEG studies. A convergent thinking test of creativity in which the participant is presented with three stimuli words and must generate a fourth association word that forms three familiar compound words or phrases.

Convergent thinking The cognitive process of generating a single, correct answer to a closed-ended problem.

Cortex The outer layer of the brain composed of neural tissue.

Cortical pyramidal cells Neurons with a triangular body and elongated apical dendrites, oriented perpendicular to the cortex.

Creativity The production of ideas that are both novel and useful in a given context.

Dendrite Cellular extensions of a neuron with many branches. Dendrites receive stimulation and transmit electrical messages to the neuron.

Dipole A separation of positive and negative electrical charges.

Divergent thinking The cognitive process of generating multiple, unique responses to an open-ended problem.

Electroencephalography A neurological technique used to record electrical activity of the brain.

Evaluation Assessing the appropriateness of the product within context. 
Event-Related Potential A specialized form of electroencephalography that measures the voltage generated by the brain in response to a specific event or stimuli. Excitatory postsynaptic potential (EPSP) A postsynaptic potential that reduces the chances of an action potential firing.

Filter model A selective auditory attention model proposed by Broadbent. Prior to information processing, auditory stimuli pass through a selective filter which evaluates the physical characteristics of the stimuli (i.e. location, pitch).

Flexibility The ability to generate a novel approach to a problem.

Fluency The ability to generate a large number of ideas in a short amount of time. High-pass filter A filter that passes frequencies higher than a specified freuency and attenuates frequencies lower than the specification.

Inhibitory postsynaptic potential (IPSP) A postsynaptic potential that reduces the chances of an action potential firing.

Inion The projection of the occipital bone at the base of the skull.

Insight A type of convergent thinking task, often defined by an Aha! moment experienced when the solver finally reaches the solution, after hitting a mental impasse.

Low-pass filter A filter that passes frequencies lower than a specified freuency and attenuates frequencies higher than the specification.

Nasion The point where the bridge of the nose meets the forehead.

Neurotransmitter A chemical messenger released from the axon terminal of a presynaptic neuron, and binds to the dendrites of a postsynaptic neuron.

Notch filter A filter that attenuates frequencies within a specified range.

Novelty The uniqueness of an idea in relation to the norm. In creativity, it can be assessed by word-association tasks. 
Postsynaptic neuron The neuron that receives the electrical impulse from the first, presynaptic neuron.

Postsynaptic potentials Changes in the membrane potential that occur when a presynaptic neuron releases neurotransmitters from the axon terminal, which then bind to the dendrites of the postsynaptic neuron.

Pre-auricular point The area where the jaw meets the skull.

Presynaptic neuron The neuron from which an electrical impulse is transmitted to the cell body of a second, postsynaptic neuron.

Remote Associates Task (RAT) A convergent thinking test of creativity based on word-associations. The participant is presented with three stimuli words and must generate a fourth word that associates with all three stimuli words.

Sensory gating The neurological process of filtering out redundant or irrelevant environmental stimuli in the brain.

Synthesizing and analyzing ability Organizing smaller ideas into broader ones, or deconstructing ideas to investigate the smaller parts. 
Appendix A

Sample task with instructions from the ATTA manual: Bold text represents verbal instructions. Regular text represents instructions for the experimenter to follow. Activity \#1

Open your booklet and fold the cover back to page one so that only Activity \#1 can be seen.

Pause. Then say:

I will now read the instructions for Activity \#1 and you may follow along with me by looking at your test booklet.

Pause. Begin to read instructions.

Just suppose you could walk on air or fly without being in an airplane or similar vehicle. What problems might this create? List as many as you can.

Pause. Then say:

You have 3 minutes to respond to this activity. Do not continue to the next activity until I tell you to do so. Ready? Begin.

Allow exactly 3 minutes. Then say:

Stop. 


\section{Appendix B}

Scoring procedures for the ATTA are based on the original Torrance Test of Creative Thinking (TTCT). They involve four norm-referenced abilities, which are: 1) Fluency, 2) Originality, 3) Elaboration, and 4) Flexibility. The ATTA manual defines these abilities as the following:

1. Fluency: the ability to produce quantities of ideas which are relevant to the task instruction.

2. Originality: the ability to produce uncommon ideas or ideas that are totally new or unique.

3. Elaboration: the ability to embellish ideas with details.

4. Flexibility: the ability to process information or objects in different ways, given the same stimulus.

These measures are scored on a raw scale, then converted to a normalized scaled score. For example, task \#3 asks the participant to elaborate on some incomplete figures (a series of triangles). The participant receives 1 point for fluency per novel drawing. If the drawn concept is similar to the norms listed in the manual, they receive a 0 for originality. Elaboration is scored for the number of details provided, and flexibility is scored for the number of unique ways that the triangles are used. Each of the three tasks are scored for fluency and originality; only the third task is scored for flexibility. The scaled normalized score allows for comparison across all four abilities, since each ability represents a different facet of creativity. Additionally, there are 15 criterion indicators. The first five are for the verbal activity and include: Richness and colourfulness of imagery; Emotions/feelings; 
Future orientation; Humour: Conceptual incongruity; Provocative questions. The last ten are for the verbal activity and include: Openness: Resistance to premature closure; Unusual visualization, different perspective; Movement and/or sound; Richness and/or colourfulness of imagery; Abstractness of titles; Context: Environment for object, articulateness in telling story; Combination/synthesis of two or more figures; Internal visual perspective; Expressions of feelings and emotions; Fantasy.

For details, see Goff and Torrance (2002). 


\section{Appendix C}

Instructions for CRA and control task presented on screen to participants.

Welcome to the experiment!

\section{[Instruction screen 1]}

This experiment involves two tasks: a WORD task and a NUMBER task.

For the WORD task, you will be shown a set of 3 words on the screen. Your goal is

to come up with a single word that combines with each word individually to create three familiar compound words or phrases.

\section{[Instruction screen 2]}

Once you have your answer, press the green button on the keypad, and at the prompt say your answer out loud. Press the green button again to go to the next trial. If you dont indicate a response, the trial will end after some time. Lets practice.

[Practice trial 1] dew comb bee

[Practice trial 2] night wrist stop

[Practice trial 3] cane daddy plum

\section{[Instruction screen 3]}

For the number task, you will again be shown 3 words. This time though, you will try to find the difference between the number of consonants and the number of vowels. Lets see an example.

[Practice trial 1] comb wrist cane [Practice trial 2] stop bee daddy [Practice trial 3] plum night dew 


\section{References}

Adler, L., Pachtman, E., Franks, R., Pecevich, M., Waldo, M., \& Freedman, R. (1982). Neurophysiological evidence for a defect in neuronal mechanisms involved in sensory gating in schizophrenia. Biological Psychiatry, 17(6), 639-654.

Amabile, T. (1996). Creativity in Context. Boulder: Westview Press.

Arden, R., Chavez, R. S., Grazioplene, R., \& Jung, R. E. (2010). Neuroimaging creativity: A psychometric view. Behavioural Brain Research, 214, 143-156. Retrieved from http://dx.doi.org/10.1016/j.bbr.2010.05.015 doi: 10.1016/j.bbr.2010.05.015

Aspinwall, L. G. (1998). Rethinking the Role of Positive Affect in Self-Regulation. Motivation and Emotion, 22(1), 1-32. Retrieved from http://search. proquest. com/docview/757986152?accountid=16482\$\ backslash\$nhttp://ra5jz3az8r. search.serialssolutions.com/

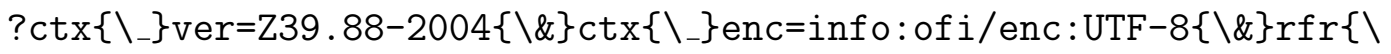
_\}id=info:sid/ProQ:abiglobal $\{\backslash \&\}$ rft $\{\backslash\}\}$ val $\left\{\backslash_{-}\right\}$fmt=info: ofi/fmt: kev:mtx: journal $\{\backslash\}$ rft.genre=article $\{\backslash \&$ rft.jti doi: http://dx.doi.org/10.1023/A:1023080224401

Barkley, R. a. (1997). Behavioral inhibition, sustained attention, and executive functions: constructing a unifying theory of ADHD. Psychological bulletin, 
121 (1), 65-94. doi: 10.1037/0033-2909.121.1.65

Barron, F., \& Harrington, D. (1981). Creativity, Intelligence, and Personality. Annual Review of Psychology. Annual Review of Psychology, 32, 439-476. doi: http://dx.doi.org/10.1146/annurev.ps.32.020181.002255

Benedek, M., Fink, A., \& Neubauer, A. C. (2006). Enhancement of Ideational Fluency by Means of Computer-Based Training. Creativity Research Journal, 18(3), 317-328. doi: 10.1207/s15326934crj1803_7

Benedek, M., \& Neubauer, A. C. (2013). Revisiting mednick's model on creativity-related differences in associative hierarchies. Evidence for a common path to uncommon thought. Journal of Creative Behavior, 47(4), 273-289. doi: $10.1002 /$ jocb.35

Boutros, N. N., Korzyukov, O., Jansen, B., Feingold, A., \& Bell, M. (2004). Sensory gating deficits during the mid-latency phase of information processing in medicated schizophrenia patients. Psychiatry Research, 126(3), 203-215. doi: 10.1016/j.psychres.2004.01.007

Bowden, E. M., \& Jung-Beeman, M. (2003). Normative data for 144 compound remote associate problems. Behavior ResearchMethods, Instruments, $\mathcal{E}$ Computers, 35(4), 634-639. doi: 10.3758/BF03195543

Bowden, E. M., Jung-beeman, M., Fleck, J., \& Kounios, J. (2005). New approaches to demystifying insight. TRENDS in Cognitive Sciences, 9(7), 322-328. doi: 10.1016/j.tics.2005.05.012

Braff, D. L., \& Geyer, M. A. (1990). Sensorimotor Gating and Schizophrenia: Human and Animal Model Studies. Archives of General Psychiatry, 47(2), $181-188$. 
Broadbent, D. E. (1958). Perception and Communication. New York: Permagon Press.

Carson, S. H., Peterson, J. B., \& Higgins, D. M. (2003). Decreased latent inhibition is associated with increased creative achievement in high-functioning individuals. Journal of Personality and Social Psychology, 85(3), 499-506. doi: 10.1037/0022-3514.85.3.499

Colzato, L. S., Ozturk, A., \& Hommel, B. (2012). Meditate to create: The impact of focused-attention and open-monitoring training on convergent and divergent thinking. Frontiers in Psychology, 3(APR), 1-5. doi: 10.3389/fpsyg.2012.00116

Dalecki, A., Johnstone, S. J., \& Croft, R. J. (2015). Clarifying the functional process represented by $\mathrm{P} 50$ suppression. International Journal of Psychophysiology, 96(3), 149-154. Retrieved from http://dx.doi.org/10.1016/j.ijpsycho.2015.04.011 doi: 10.1016/j.ijpsycho.2015.04.011

De Dreu, C. K. W., Nijstad, B. A., Baas, M., Wolsink, I., \& Roskes, M. (2012). Working Memory Benefits Creative Insight, Musical Improvisation, and Original Ideation Through Maintained Task-Focused Attention. Personality and Social Psychology Bulletin, 38(5), 656-669. Retrieved from http://psp.sagepub.com/cgi/doi/10.1177/0146167211435795 doi: $10.1177 / 0146167211435795$

Delorme, A., Palmer, J., Oostenveld, R., Onton, J., \& Makeig, S. (2009). More independent EEG components tend to be more dipolar. In Organization for human brain mapping 2009 annual meeting (p. S123). 
Dickter, C. L., \& Kieffaber, P. D. (2014). EEG Methods for the Psychological Sciences (M. Carmichael, Ed.). Chennai: SAGE Publications.

Dietrich, A., \& Kanso, R. (2010). A Review of EEG , ERP , and Neuroimaging Studies of Creativity and Insight. Psychological Bulletin, 136(5), 822-848. doi: $10.1037 / \mathrm{a} 0019749$

Dolu, N., Süer, C., \& Özesmi, Ç. (2001). A comparison of the different interpair intervals in the conditioning-testing P50 paradigms. International Journal of Psychophysiology, 41(3), 265-270. doi: 10.1016/S0167-8760(01)00134-9

Domino, G. (1970). Identification of potentially creative persons from the Adjective Check List. Journal of Consulting and Clinical Psychology, 1, 48-51.

Donchin, E., Ritter, W., \& McCallum, W. C. (1978). Cognitive psychophysiology: The endogenous components of the ERP. In E. Callaway, P. Tueting, \& S. Hoslow (Eds.), Event-related brain potentials in man (pp. 349-441). New York: Academic Press.

Dorfman, L., \& Gassimova, V. (2015). Divergent thinking and differential focusing of perceptual attention in visual serial search tasks. Learning and Individual Differences, 44, 25-32. Retrieved from http://dx.doi.org/10.1016/j.lindif.2015.05.007 doi: 10.1016/j.lindif.2015.05.007

Eysenck, H. (1995). Genius: The natural history of creativity. Cambridge: Cambridge University Press.

Feist, G., \& Barron, F. (2003). Predicting creativity from early to late adulthood: intellect, potential, and personality. Journal of Research Personality, 37, 62-88. 
Fink, A., \& Benedek, M. (2014). EEG alpha power and creative ideation. Neuroscience and Biobehavioural Reviews, 44, 111-123.

Fink, A., Grabner, R. H., Benedek, M., Reishofer, G., Hauswirth, V., Fally, M., ... Neubauer, A. C. (2009). The creative brain: Investigation of brain activity during creative problem solving by means of EEG and fMRI. Human Brain Mapping, 30(3), 734-748. doi: 10.1002/hbm.20538

Fink, A., Graif, B., \& Neubauer, A. C. (2009). Brain correlates underlying creative thinking: EEG alpha activity in professional vs . novice dancers. NeuroImage, 46(3), 854-862. Retrieved from

http://dx.doi.org/10.1016/j.neuroimage.2009.02.036 doi:

10.1016/j.neuroimage.2009.02.036

Fink, A., \& Neubauer, A. C. (2006). EEG alpha oscillations during the performance of verbal creativity tasks: Differential effects of sex and verbal intelligence. International Journal of Psychophysiology, 62(1), 46-53. doi: 10.1016/j.ijpsycho.2006.01.001

Fink, A., Schwab, D., \& Papousek, I. (2011). Sensitivity of EEG upper alpha activity to cognitive and affective creativity interventions. International Journal of Psychophysiology, 82(3), 233-239. Retrieved from http://dx.doi.org/10.1016/j.ijpsycho.2011.09.003 doi:

10.1016/j.ijpsycho.2011.09.003

Finke, R. (1995). Creative insight and preinventive forms. In R. J. Sternberg \& J. Davidson (Eds.), The nature of insight (1st paperb ed., pp. 255-280). Cambridge: MIT Press.

Fiore, S., Schooler, J., Linville, P., \& Hasher, L. (2001). The creative costs and 
benefits of inhibition. Abstracts of the Psychonomic Society, 6(42).

Fredrickson, B. L. (2001). The Role of Positive Emotions in Positive Psychology. American Psychologist, 56(3), 218-226. doi: 10.1037/0003-066X.56.3.218

Freedman, R., Adler, L., Waldo, M., Pachtman, E., \& Franks, R. (1983).

Neurophysiological evidence for a defect in inhibitory pathways in schizophrenia: comparison of medicated and drug-free patients. Biological Psychiatry, 18(5), 537-551.

Friedman, R. S., Förster, J., \& Denzler, M. (2007). Interactive Effects of Mood and Task Framing on Creative Generation. Creativity Research Journal, 19(2-3), 141-162. doi: 10.1080/10400410701397206

Gardner, H. (1983). Frames of Mind: The Theory of Multiple Intelligences. New York: Basic Books.

Gaudreault, P. O., Gagnon, J. F., Montplaisir, J., Vendette, M., Postuma, R. B., Gagnon, K., \& Gosselin, N. (2013). Abnormal occipital event-related potentials in Parkinson's disease with concomitant REM sleep behavior disorder. Parkinsonism and Related Disorders, 19(2), 212-217. Retrieved from http://dx.doi.org/10.1016/j.parkreldis.2012.10.006 doi: 10.1016/j.parkreldis.2012.10.006

Ghiselin, B., Rompel, R., \& Taylor, C. (1964). A creative process checklist: Its development and validation (C. Taylor, Ed.).

Glover, J. A., Reynolds, C., \& Ronning, R. (Eds.). (1989). Handbook of Creativity: Perspectives on individual differences. New York: Plenum.

Goff, K., \& Torrance, E. P. (2002). Abbreviated Torrance Test for Adults Manual. Bensenville: Scholastic Testing Service, Inc. 
Grabner, R. H., Fink, A., \& Neubauer, A. C. (2007). Brain Correlates of Self-Rated Originality of Ideas: Evidence From Event-Related Power and Phase-Locking Changes in the EEG. Behvaioural Neuroscience, 121(1), 224-230. doi: 10.1037/0735-7044.121.1.224

Groborz, M., \& Necka, E. (2003). Creativity and Cognitive Control: Explorations of Generation and Evaluation Skills. Creativity Research Journal, 15, 183-197.

Guilford, J. P. (1950). Creativity. American Psychologist, 5(9), 444-454.

Guterman, Y., Josiassen, R., \& Bashore, T. J. (1992). Attentional influence on the P50 component of the auditory eventrelated brain potential. International Journal of Psychophysiology, 12(2), 197-209.

Hennessey, B. (2004). Developing Creativity in Gifted Children: The Central Importance of Motivation and Classroom Climate. National Research Centre for the Gifted and Talented.

Hennessey, B. A., \& Amabile, T. M. (2010). Creativity. Annual Review of Psychology, 61(1), 569-598. Retrieved from http:// www . annualreviews.org/doi/abs/10.1146/annurev .psych.093008.100416 doi: 10.1146/annurev.psych.093008.100416

Hillyard, S. A., Hink, R. F., Schwent, V. L., \& Picton, T. W. (1973). Electrical Signs of Selective Attention in the Human Brain. American Association for the Advancement of Science, 182, 177-180.

Hocevar, D. (1979). Ideational fluency as a confounding factor in the measurement of originality. Journal of Educational Psychology, 71(2), 191-196. Retrieved from http://content.apa.org/journals/edu/71/2/191 doi: $10.1037 / / 0022-0663.71 .2 .191$ 
Isen, A. M., \& Reeve, J. (2005). The influence of positive affect on intrinsic and extrinsic motivation: Facilitating enjoyment of play, responsible work behavior, and self-control. Motivation and Emotion, 29(4), 297-325. doi: $10.1007 / \mathrm{s} 11031-006-9019-8$

Jauk, E., Benedek, M., \& Neubauer, A. C. (2012). Tackling creativity at its roots: Evidence for different patterns of EEG alpha activity related to convergent and divergent modes of task processing. International Journal of Psychophysiology, 84(2), 219-225. Retrieved from http://dx.doi.org/10.1016/j.ijpsycho.2012.02.012 doi: 10.1016/j.ijpsycho.2012.02.012

Jerger, K., Biggins, C., \& Fein, G. (1992). P50 suppression is not affected by attentional manipulations. Biological Psychiatry, 31(4), 365-377. doi: 10.1016/0006-3223(92)90230-W

Kahneman, D. (1973). Attention and Effort. Englewood Cliffs, New Jersey: Prentice-Hall Inc.

Kasof, J. (1997). Creativity and Breadth of Attention. Creativity Research Journal, 10(4), 303-315. doi: 10.1207/s15326934crj1004

Kaufmann, G. (2003). Expanding the mood - Creativity equation. Creativity Research Journal, 15(2-3), 131-135. Retrieved from $\{\backslash$ textless\}GotoISI $\{\backslash$ textgreater $\}: / / 000185171200003$ doi: 10.1207/S15326934CRJ152

Kim, K. H. (2006). Can We Trust Creativity Tests? A Review of the Torrance Tests of Creative Thinking (TTCT). Creativity Research Journal, 18(1), 3-14. doi: 10.1207/s15326934crj1801_2 
Klimesch, W. (1999). EEG alpha and theta oscillations reflect cognitive and memory performance: A review and analysis. Brain Research Reviews, 29(2-3), 169-195. doi: 10.1016/S0165-0173(98)00056-3

Klimesch, W., Sauseng, P., Hanslmayr, S., Gruber, W., \& Freunberger, R. (2007). Event-related phase reorganization may explain evoked neural dynamics. Neuroscience and Biobehavioral Reviews, 31(7), 1003-1016. doi: 10.1016/j.neubiorev.2007.03.005

Kris, E. (1952). Psychoanalytic explorations in art. New York: International Universities Press.

Kumar, G. (1978). No Creativity functioning ni relation to personality, value-orientation and achievement motivation. Indian Educational Review, $13,110-115$.

Kuperberg, G. R. (2004). Electroencephalography, Event-Related Potentials, and Magnetoencephalography. In D. D. Dougherty, S. L. Rauch, \& J. F. Rosenbaum (Eds.), Essentials of neuroimaging for clinical practice (pp. 117-127). American Psychiatric Pub.

Landa, L., Krpoun, Z., Kolarova, M., \& Kasparek, T. (2014). Event-related potentials and their applications. ANS: Journal for Neurocognitive Research, $56(1), 17-23$.

Lang, S., Kanngieser, N., Jaśkowski, P., Haider, H., Rose, M., \& Verleger, R. (2006). Precursors of insight in event-related brain potentials. Journal of cognitive neuroscience, 18(12), 2152-66. Retrieved from http://www.ncbi.nlm.nih.gov/pubmed/17129197 doi: 10.1162/jocn.2006.18.12.2152 
Lavric, A., Forstmeier, S., \& Rippon, G. (2000). Differences in working memory involvement in analytical and creative tasks: an ERP study. Cognitive Neuroscience, 11(8), 1613-1618. doi: 10.1097/00001756-200006050-00004

Light, G. A., \& Braff, D. L. (2003). Sensory gating deficits in schizophrenia: Can we parse the effects of medication, nicotine use, and changes in clinical status. Clinical Neuroscience Research, 3, 47-54. doi: 10.1016/S1566-2772(03)00018-5

Lijffijt, M., Moeller, F. G., Boutros, N. N., Steinberg, J. L., Meier, S. L., Lane, S. D., \& Swann, A. C. (2009). Diminished P50, N100 and P200 auditory sensory gating in bipolar I disorder. Psychiatry Research, 167(3), 191-201. doi: 10.1016/j.psychres.2008.04.001.Diminished

Luck, S. (2014). An Introduction to the Event-Related Potential Technique (2nd ed.). Massachusetts: MIT Press.

Luck, S., \& Kappenman, E. (2011). The Oxford Handbook of Event-Related Potential Components (S. Luck \& E. Kappenman, Eds.).

Luck, S. J., \& Hillyard, S. a. (1994). Spatial filtering during visual search: Evidence from human electrophysiology. Journal of Experimental Psychology: Human Perception and Performance, 20(5), 1000-1014. Retrieved from http://www.ncbi.nlm.nih.gov/pubmed/7964526 doi:

10.1037/0096-1523.20.5.1000

MacKinnon, D. (1970). The personality correlates of Creativity: A student of American architects. New York: Penguin.

Mai, X. Q., Luo, J., Wu, J. H., \& Luo, Y. J. (2004). "Aha!" Effects in a guessing riddle task: An event-related potential study. Human Brain Mapping, 22(4), 
261-270. doi: 10.1002/hbm.20030

Martin, L. L., Ward, D. W., Achee, J. W., \& Wyer, R. S. (1993). Mood as input:

People have to interpret the motivational implications of their moods. Journal of Personality and Social Psychology, 64(3), 317-326. doi:

10.1037/0022-3514.64.3.317

Martindale, C. (1999). Biological basis of creativity. In R. J. Sternberg (Ed.), 1handbook of creativity (pp. 137-152). New York: Cambridge University Press.

Martindale, C., \& Hasenfus, N. (1978). EEG differences as a function of creativity, stage of the creative process and effort to be original. Biological Psychology, 6, 157-167. doi: 10.1017/CBO9781107415324.004

Martindale, C., \& Hines, D. (1975). Creativity and cortical activation during creative, intellectual and eeg feedback tasks. Biological Psychology, 3(2), 91-100. doi: 10.1016/0301-0511(75)90011-3

Martindale, C., Hines, D., Mitchell, L., \& Covello, E. (1984). EEG alpha asymmetry and creativity. Personality and Individual Differences, 5(1), 77-86. doi: 10.1016/0191-8869(84)90140-5

Mednick, S. (1962). The associative basis of the creative process. Psychological Review, 69(3), 220-232.

Mendelsohn, G. (1976). Associative and attentional processes in creative performance. Journal of Personality, 44, 341-369. Retrieved from http://doi.wiley.com/10.1111/j.1467-6494.1976.tb00127.x doi: 10.1111/j.1467-6494.1976.tb00127.x

Mendelsohn, G., \& Griswold, B. (1964). Differential use of incidental stimuli in 
problem solving as a function of creativity. Journal of Abnormal and Social Psychology, 68, 431-436.

Mendelsohn, G., \& Lindholm, E. (1972). Individual differences and the role of attention in the use of cues in verbal problem solving '. Journal of Personality, 40(2), 226-241.

Mikyska, C. (2012). Auditory Selective Attention: An introduction and evidence for distinct facilitation and inhibition mechanisms (Unpublished doctoral dissertation). University of California.

Näätänen, R., \& Picton, T. (1987). Näätänen1987.pdf. Psychophysiology, 24(4), $375-425$.

Necka, E. (1999). Creativity and attention. Psychological Bulletin, 30, 85-98.

Pfenninger, K., \& Shubik, V. (2001). The Origins of Creativity. New York: Oxford University Press.

Picton, T. W., \& Hillyard, S. a. (1974). Human auditory evoked potentials II: Effects of attention. Electroencephalography and Clinical Neurophysiology, 36, 191-199. doi: 10.1016/0013-4694(74)90156-4

Powers, P., Stevens, C., \& Andreasen, N. (1975). The ontogenesis of intelligence: Evaluating the Piaget theory. Comprehensive Psychiatry, 16(2), 149-154.

Qiu, J., Li, H., Jou, J., Wu, Z., \& Zhang, Q. (2008). Spatiotemporal cortical activation underlies mental preparation for successful riddle solving: An event-related potential study. Experimental Brain Research, 186(4), 629-634. doi: $10.1007 / \mathrm{s} 00221-008-1270-7$

Qiu, J., Li, H., Yang, D., Luo, Y., Li, Y., Wu, Z., \& Zhang, Q. (2008). The neural basis of insight problem solving: An event-related potential study. Brain and 
Cognition, 68(1), 100-106. doi: 10.1016/j.bandc.2008.03.004

Qiu, J., Luo, Y. J., Wu, Z., \& Zhang, Q. (2006). A further study of ERP effects of "insight" in a riddle guessing task. Acta Psychologica Sinica, 38, 507-514.

Rawlings, D. (1985). Psychoticism, creativity and dichotic shadowing. Personality and Individual Differences, 6(6), 737-742. doi: 10.1016/0191-8869(85)90084-4

Razumnikova, O. M. (2007). Creativity related cortex activity in the remote associates task. Brain Research Bulletin, 73, 96-102. doi: 10.1016/j.brainresbull.2007.02.008

Rentzsch, J., Jockers-Scherübl, M. C., Boutros, N. N., \& Gallinat, J. (2008). Test-retest reliability of P50, N100 and P200 auditory sensory gating in healthy subjects. International Journal of Psychophysiology, 67(2), 81-90. doi: 10.1016/j.ijpsycho.2007.10.006

Runco, M. A., \& Albert, R. S. (1985). The reliability and validity of ideational originality in the divergent thinking of academically gifted and nongifted children. Educational and Psychological Measurement, 45(3), 483-501. doi: $10.1177 / 001316448504500306$

Runco, M. A., \& Mraz, W. (1992). from the SAGE Social Science Collections . All Rights. Educational and Psychological Measurement, 52, 213-221. doi: 0803973233

Rutter, B., Kröger, S., Hill, H., Windmann, S., Hermann, C., \& Abraham, A. (2012). Can clouds dance? Part 2: An ERP investigation of passive conceptual expansion. Brain and Cognition, 80(3), 301-310. Retrieved from http://dx.doi.org/10.1016/j.bandc.2012.08.003 doi: 
10.1016/j.bandc.2012.08.003

Simonton, D. (1999). Origins of Genius: Darwinian Perspectives on Creativity. New York: Oxford University Press.

Smith, D. A., Boutros, N. N., \& Schwarzkopf, S. B. (1994). Reliability of P50 auditory event-related potential indices of sensory gating. Psychophysiology, 31 (5), 495-502. doi: 10.1111/j.1469-8986.1994.tb01053.x

Srinivasan, N. (2007). Cognitive neuroscience of creativity: EEG based approaches. Methods, 42, 109-116. doi: 10.1016/j.ymeth.2006.12.008

Sternberg, R. J. (2005). Creativity or creativities? International Journal of Human Computer Studies, 63(4), 370-382. doi: 10.1016/j.ijhcs.2005.04.003

Sternberg, R. J., \& Lubart, T. (1995). Defying the Crowd: Cultivating Creativity in a Culture of Conformity. New York: Free Press.

Sur, S., \& Sinha, V. (2009). Event-related potential: An overview. Industrial Psychiatry, 18(1), 70.

Tapasak, R., Roodin, P., \& Vaught, G. (1978). Effects of extraversion, anxiety, and sex on children's verbal fluency and coding task performance. Journal of Psychology, 100, 49-55. doi: 10.1017/CBO9781107415324.004

Treisman, A. (1964). Selective attention in man. British Medical Bulletin, 20, $12-16$.

Vosburg, S. (1998). The effects of positive and negative mood on divergent-thinking performance. Creativity Research Journal, 11(January), 165-172. Retrieved from

http://www.tandfonline.com/doi/abs/10.1207/s15326934crj1102\{\_\}6

doi: $10.1207 / \mathrm{s} 15326934 \mathrm{crj} 1102$ 
Weisberg, R. (1986). Creativity, Genius and Other Myths. Freeman.

Weisberg, R. (1999). Creativity and knowledge: A challenge to the theories. In R. J. Sternberg (Ed.), Handbook of creativity (pp. 226-250). New York: Cambridge University Press.

White, H. A., \& Shah, P. (2006). Uninhibited imaginations: Creativity in adults with Attention-Deficit/Hyperactivity Disorder. Personality and Individual Differences, 40(6), 1121-1131. doi: 10.1016/j.paid.2005.11.007

Winner, E. (2000). The origins and ends of giftedness. American Psychologist, 55(1), 159-169. doi: 10.1037/0003-066X.55.1.159

Woldorff, M. G., Hansen, J. C., \& Hillyard, S. A. (1987). Evidence for effects of selective attention in the mid-latency range of the human auditory event-related potential. Electroencephalography and Clinical Neurophysiology, $40,146-154$.

Woldorff, M. G., \& Hillyard, S. A. (1991). Modulation of early auditory processing during selective listening to rapidly presented tones. Electroencephalography and Clinical Neurophysiology, 79(3), 170-191. doi: 10.1016/0013-4694(91)90136-R

Zabelina, D. L., O'Leary, D., Pornpattananangkul, N., Nusslock, R., \& Beeman, M. (2015). Creativity and sensory gating indexed by the P50: Selective versus leaky sensory gating in divergent thinkers and creative achievers. Neuropsychologia, 69, 77-84. Retrieved from http://dx.doi.org/10.1016/j.neuropsychologia.2015.01.034 doi: 10.1016/j.neuropsychologia.2015.01.034

Zabelina, D. L., \& Robinson, M. D. (2010). Creativity as flexible cognitive control. 
Psychology of Aesthetics, Creativity and the Arts, 4(3), 136-143. doi: $10.1037 / \mathrm{a} 0017379$

Zouridakis, G., \& Boutros, N. N. (1992). Stimulus parameter effects on the P50 evoked response. Biological Psychiatry, 32, 839-841. doi:

10.1016/0006-3223(92)90088-H 